\title{
DYNAMICS AND STATICS OF FLEXIBLE AXIALLY SYMMETRIC SHALLOW SHELLS
}

\author{
J. AWREJCEWICZ, V. A. KRYSKO, AND I. V. KRAVTSOVA
}

Received 24 June 2005; Accepted 12 July 2005

In this work, we propose the method for the investigation of stochastic vibrations of deterministic mechanical systems represented by axially symmetric spherical shells. These structure members are widely used as sensitive elements of pressure measuring devices in various branches of measuring and control industry, machine design, and so forth. The proposed method can be easily extended for the investigation of shallow spherical shells, goffer-type membranes, and so on. The so-called charts of control parameters for a shell subjected to a transversal uniformly distributed and local harmonic loading force and resistance moment are constructed. The scenarios of the transition of vibration of shallowtype system into chaotic state are investigated with the use of the theory of differential equations and the theory of nonlinear dynamics. The method of the control of chaotic vibrations of flexible spherical shells subjected to a transversal harmonic load through a synchronized action of either harmonic resistance moment or force is proposed, illustrated, and discussed.

Copyright (c) $2006 \mathrm{~J}$. Awrejcewicz et al. This is an open access article distributed under the Creative Commons Attribution License, which permits unrestricted use, distribution, and reproduction in any medium, provided the original work is properly cited.

\section{Introduction}

Stochastic vibrations of flexible shallow shells are rather rarely investigated. It is mainly static problems as well as those of dynamical stability loss subjected to impulse time loads constant in time that is the subject of investigation, and hence the similar problems but subjected to a harmonic transversal load are not often studied. A reason for that is the fact that the general theory of nonlinear dissipative mechanical systems is not yet fully developed. We focus our attention on filling the gap occurring in this field. It is worth noticing that the investigation of vibrations of flexible plates and shells has been recently initiated $[1-8,15,17,18]$.

On the other hand, the control of chaos in deterministic systems belongs to difficult and challenging tasks of nonlinear dynamical systems investigation, since chaotic

Hindawi Publishing Corporation

Mathematical Problems in Engineering

Volume 2006, Article ID 35672, Pages 1-25

DOI 10.1155/MPE/2006/35672 
vibrations are relatively often exhibited by various engineering, economic, and biological systems.

Firstly, the problem of chaos control has been stated in [12-14] and in the classical work of Ott et al. [22]. In [26] one may find even earlier sources of this idea. Problems related to the control of chaos have been considered in hydrodynamics [27], chemistry [23], and biology and medicine [25]. However, this problem has not been investigated in the theory of shells, at least to the authors' knowledge.

\section{Problem formulation and computational algorithm}

Consider a spherical axially symmetric shallow shell creating a closed two-dimensional subspace of space $R^{2}$ in the polar coordinate system introduced in the following manner: $\Omega=\{(r, z) \mid r \in[0, b],-h / 2 \leq z \leq h / 2\}$. Equations governing the dynamics of shallow axially symmetric shells are cast in the form [29]

$$
\begin{gathered}
w^{\prime \prime}+\varepsilon w^{\prime}=-\frac{\partial^{4} w}{\partial r^{4}}-\frac{2}{r} \frac{\partial^{3} w}{\partial r^{3}}+\frac{1}{r^{2}} \frac{\partial^{2} w}{\partial r^{2}}-\frac{1}{r^{3}} \frac{\partial w}{\partial r}-\frac{\phi}{r}\left(1-\frac{\partial^{2} w}{\partial r^{2}}\right)-\frac{\partial \phi}{\partial r}\left(1-\frac{1}{r} \frac{\partial w}{\partial r}\right)+4 q, \\
\frac{\partial^{2} \phi}{\partial r^{2}}+\frac{1}{r} \frac{\partial \phi}{\partial r}-\frac{1}{r^{2}} \frac{\partial \phi}{\partial r}=\frac{\partial w}{\partial r}\left(1-\frac{1}{2 r} \frac{\partial w}{\partial r}\right),
\end{gathered}
$$

where $\phi=\partial F / \partial r$. The nondimensional quantities follow: $\bar{t}=\omega_{0} t ; \omega_{0}=\sqrt{E g / \gamma R^{2}} ; \bar{\varepsilon}=$ $\sqrt{(g / \gamma E)}(R / h) \varepsilon ; \bar{F}=\eta\left(F / E h^{3}\right) ; \bar{w}=\sqrt{\eta}(w / h) ; \bar{r}=b(r / c) ; \bar{q}=\bar{q}_{3}=(\sqrt{\eta} / 4)\left(q_{3} / E\right)(R / h)^{2} ;$ $\eta=12\left(1-v^{2}\right) ; b=\sqrt[4]{\eta}(c / \sqrt{R h})$, where $t$ is the time; $\varepsilon$ is the damping coefficient of the shell surrounding medium, $F$ is the stress function, $w$ is the function of displacements; $R$, $c$ are the main and secondary radii of the curvature of supported contour, respectively; $h$ is the shell thickness; $b$ is the sloping parameter; $v$ is Poisson's coefficient; $r$ is the distance from a rotation axis to a point lying in the shell's mean surface; $q$ is the parameter of external load; $\omega_{0}$ is the frequency of linear vibrations. To simplify, bars over nondimensional quantities are omitted in (2.1). Derivatives with respect to time are denoted by ('). One has to add boundary and initial conditions to the system (2.1), as well as conditions in the shell vertex. For a ball-type movable support in the meridian direction, the boundary conditions read as

(1) (a) homogeneous boundary conditions

$$
\phi=w=0, \quad \frac{\partial^{2} w}{\partial r^{2}}+\frac{\nu}{r} \frac{\partial w}{\partial r}, \quad \text { for } r=b
$$

(b) nonhomogeneous boundary conditions

$$
\phi=w=0, \quad \frac{\partial^{2} w}{\partial r^{2}}=M_{0} \sin \left(\omega_{p} t\right), \quad \text { for } r=b
$$

(2) (a) homogeneous boundary conditions for a ball-type unmovable support

$$
\frac{\partial \phi}{\partial r}-\nu \frac{\phi}{b}=0, \quad w=0, \quad \frac{\partial^{2} w}{\partial r^{2}}+\frac{\nu}{r} \frac{\partial w}{\partial r}=0, \quad \text { for } r=b ;
$$


(b) nonhomogeneous boundary conditions

$$
\frac{\partial \phi}{\partial r}-\nu \frac{\phi}{b}=0, \quad w=0, \quad \frac{\partial^{2} w}{\partial r^{2}}+\frac{\nu}{r} \frac{\partial w}{\partial r}=M_{0} \sin \left(\omega_{p} t\right), \quad \text { for } r=b ;
$$

(3) a movable clamped (resistance) contour

$$
\phi=w=0, \quad \frac{\partial w}{\partial r}=0, \quad \text { for } r=b ;
$$

(4) an unmovable clamped (resistance) contour

$$
\frac{\partial \phi}{\partial r}-v \frac{\phi}{b}=0, \quad w=0, \quad \frac{\partial w}{\partial r}=0, \quad \text { for } r=b
$$

The initial conditions read as

$$
w=f_{1}(r, 0)=0, \quad w^{\prime}=f_{2}(r, 0)=0, \quad 0 \leq t<\infty .
$$

In the close vicinity of the shell vertex, the following estimations hold:

$$
\phi \approx A r, \quad \phi^{\prime} \approx A, \quad w \approx B+C r^{2}, \quad w^{\prime} \approx 2 C r, \quad w^{\prime \prime} \approx 2 C, \quad w^{\prime \prime \prime} \approx 0 .
$$

In order to reduce the distributed system (2.1)-(2.9) to a lumped one, the method of finite differences with approximation $O\left(\Delta^{2}\right)$ is applied. Let us express the system (2.1)(2.9) via finite-difference relations with respect to the spatial variable $r$ :

$$
\begin{aligned}
& w_{i}^{\prime \prime}+\varepsilon w_{i}^{\prime}=-\frac{w_{i+1}-w_{i-1}}{2 \Delta}\left(\frac{1}{r_{i}^{3}}-\frac{\phi_{i+1}-\phi_{i-1}}{2 r_{i} \Delta}\right)+\frac{w_{i+1}-2 w_{i}+w_{i-1}}{r_{i} \Delta^{2}}\left(\phi_{i}+\frac{1}{r_{i}}\right) \\
&-\frac{\phi_{i+1}-\phi_{i-1}}{2 \Delta}-\frac{\phi_{i}}{r_{i}}-\frac{w_{i+2}-4 w_{i+1}+6 w_{i}-4 w_{i-1}+w_{i-2}}{\Delta^{4}} \\
&-\frac{w_{i+2}-2 w_{i+1}+2 w_{i-1}-w_{i-2}}{r_{i} \Delta^{3}}+4 q_{i}, \\
& \phi_{i+1}\left(-\frac{1}{\Delta^{2}}-\frac{1}{2 r_{i} \Delta}\right)+\phi_{i}\left(\frac{2}{\Delta^{2}}+\frac{1}{r_{i}^{2}}\right)+\phi_{i-1}\left(-\frac{1}{\Delta^{2}}+\frac{1}{2 r_{i} \Delta}\right) \\
&=-\frac{w_{i+1}-w_{i-1}}{2 \Delta}\left(1-\frac{w_{i+1}-w_{i-1}}{4 r_{i} \Delta}\right),
\end{aligned}
$$

where $\Delta=b / n, n$ is a member of distributing parts of two shell radii. The boundary conditions have the following form now:

(1) (a) homogeneous boundary conditions

$$
\phi_{n}=0, \quad w_{i+1}=\frac{\nu \Delta-2 b}{2 b+v \Delta} w_{i-1}, \quad w_{n}=0 \quad \text { for } r_{n}=b ;
$$


(b) nonhomogeneous boundary conditions

$$
\phi_{n}=0, \quad w_{i+1}=\frac{M_{0} \sin \left(\omega_{p} t\right)-\left(1 / \Delta^{2}-v / 2 b \Delta\right) w_{i-1}}{\left(1 / \Delta^{2}+v / 2 b \Delta\right)}, \quad w_{n}=0 \quad \text { for } r_{n}=b
$$

(2) (a) homogenous boundary conditions for a ball-type unmovable support

$$
\phi_{i+1}=\phi_{i-1}+\frac{2 \Delta \nu}{b} \phi_{i}, \quad w_{i+1}=\frac{v \Delta-2 b}{2 b+v \Delta} w_{i-1}, \quad w_{n}=0 \quad \text { for } r_{n}=b
$$

(b) nonhomogeneous boundary conditions

$$
\begin{aligned}
& \phi_{i+1}=\phi_{i-1}+\frac{2 \Delta v}{b} \phi_{i} \\
& w_{i+1}=\frac{M_{0} \sin \left(\omega_{p} t\right)-\left(1 / \Delta^{2}-v / 2 b \Delta\right) w_{i-1}}{\left(1 / \Delta^{2}+v / 2 b \Delta\right)}, \quad w_{n}=0 \quad \text { for } r_{n}=b ;
\end{aligned}
$$

(3) a movable clamped (resistance) contour

$$
\phi_{n}=0, \quad w_{n+1}=w_{n-1}, \quad w_{n}=0 \quad \text { for } r_{n}=b
$$

(4) an unmovable clamped (resistance) contour

$$
\phi_{i+1}=\phi_{i-1}+\frac{2 \Delta v}{b} \phi_{i}, \quad w_{n+1}=w_{n-1}, \quad w_{n}=0 \quad \text { for } r_{n}=b
$$

The initial conditions have the form

$$
w_{n}=f_{1}\left(r_{k}, 0\right)=0, \quad w_{n}^{\prime}=f_{2}\left(r_{k}, 0\right)=0 \quad(0 \leq k \leq n), \quad 0 \leq t<\infty .
$$

If one neglects some small terms, after the change of differential operators by central finite-difference ones for $r=\Delta$, the following conditions hold in the shell vertex:

$$
\phi_{0}=\phi_{2}-2 \phi_{1}, \quad w_{0}=\frac{4}{3} w_{1}-\frac{1}{3} w_{2}, \quad w_{-1}=\frac{8}{3} w_{1}-\frac{8}{3} w_{2}+w_{3}
$$

Although the applied load can be changed in an arbitrary manner, in this work only either uniformly distributed load or sinusoidal $\left(q=q_{0} \sin \left(\omega_{p} t\right)\right.$, where $\omega_{p}=2 \pi / T, T$ is the period of vibration) force is analysed for boundary conditions (2.11), (2.13), (2.15), and (2.16). If sinusoidal resistance moment (2.12), (2.14) occurs, then, on the shell surface we take $q=0$.

After reduction of the problem (2.1)-(2.9) into a normal form, the associated Cauchy problem is solved using the 4 th-order Runge-Kutta method. Time step is defined by the conditions of solution stability $\left(\Delta t=3.90625 \cdot 10^{-3}\right)$. 


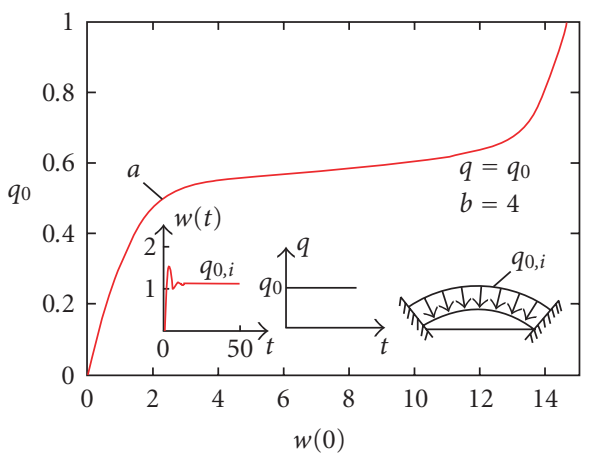

(a)

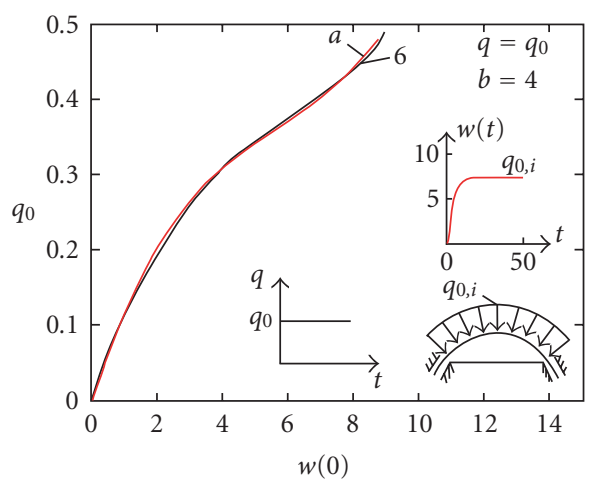

(c)

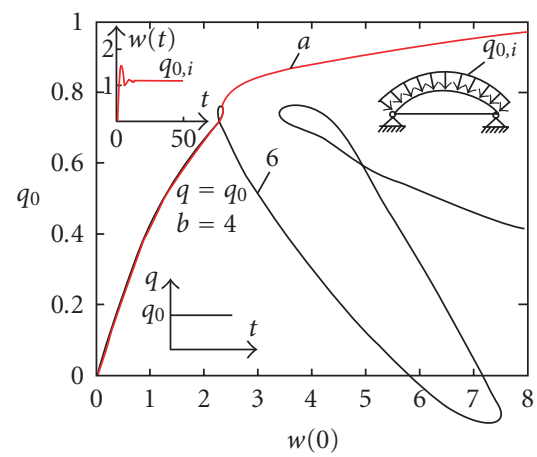

(b)

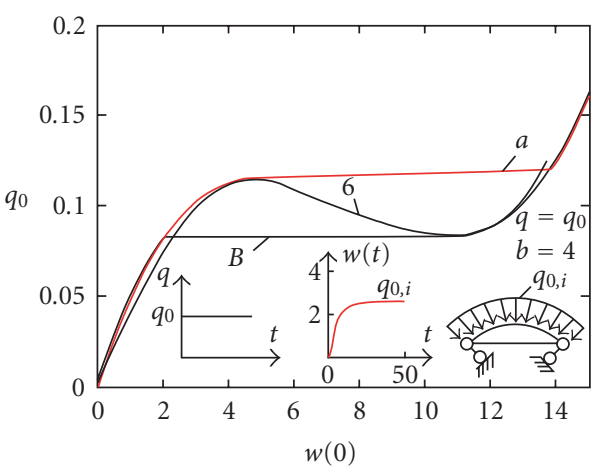

(d)

Figure 3.1. (a) Shell with a stiff clamped contour $\left(q=q_{0}, \varepsilon=1\right)$. (b) Shell with a ball-type unmovable contour $\left(q=q_{0}, \varepsilon=1\right)$. (c) Shell with a movable contour $\left(q=q_{0}, \varepsilon=1\right)$. (d) Shell with a ball-type movable contour $\left(q=q_{0}, \varepsilon=1\right)$.

\section{Reliability of results: static solution of shells stability}

The developed algorithm and program package allows to solve both static and dynamic problems. In order to solve static problems, the so-called "setup" method proposed by Fedos'ev $[9,10]$ is used. The main idea of this method follows. For $\varepsilon=\varepsilon_{c r}$, the dependency $\left\{q_{m}, w_{m}(t)\right\}, m=1,2, \ldots$, denotes numbers of loading values for which a solution has been found with the use of the setup method. Applying this method, one may compute the characteristics $q(w)$ and investigate the stress-strain state of shells. The analysis of the applied iterational setup method is given in [3]. In Figures 3.1(a)-3.1(d), the dependencies $q(w)$ obtained via both our algorithm and the Valishvili [29] method are reported for the boundary conditions shown in the bottom-right corner of each figure and for the given parameter $b$. Curves denoted by (a) correspond to our method, whereas those denoted by (b) have been obtained by Valishvili [29]. It is worth noticing that the solution obtained by Valishvili and shown in Figure 3.1(b) is not unique with respect to both load 
Table 3.1

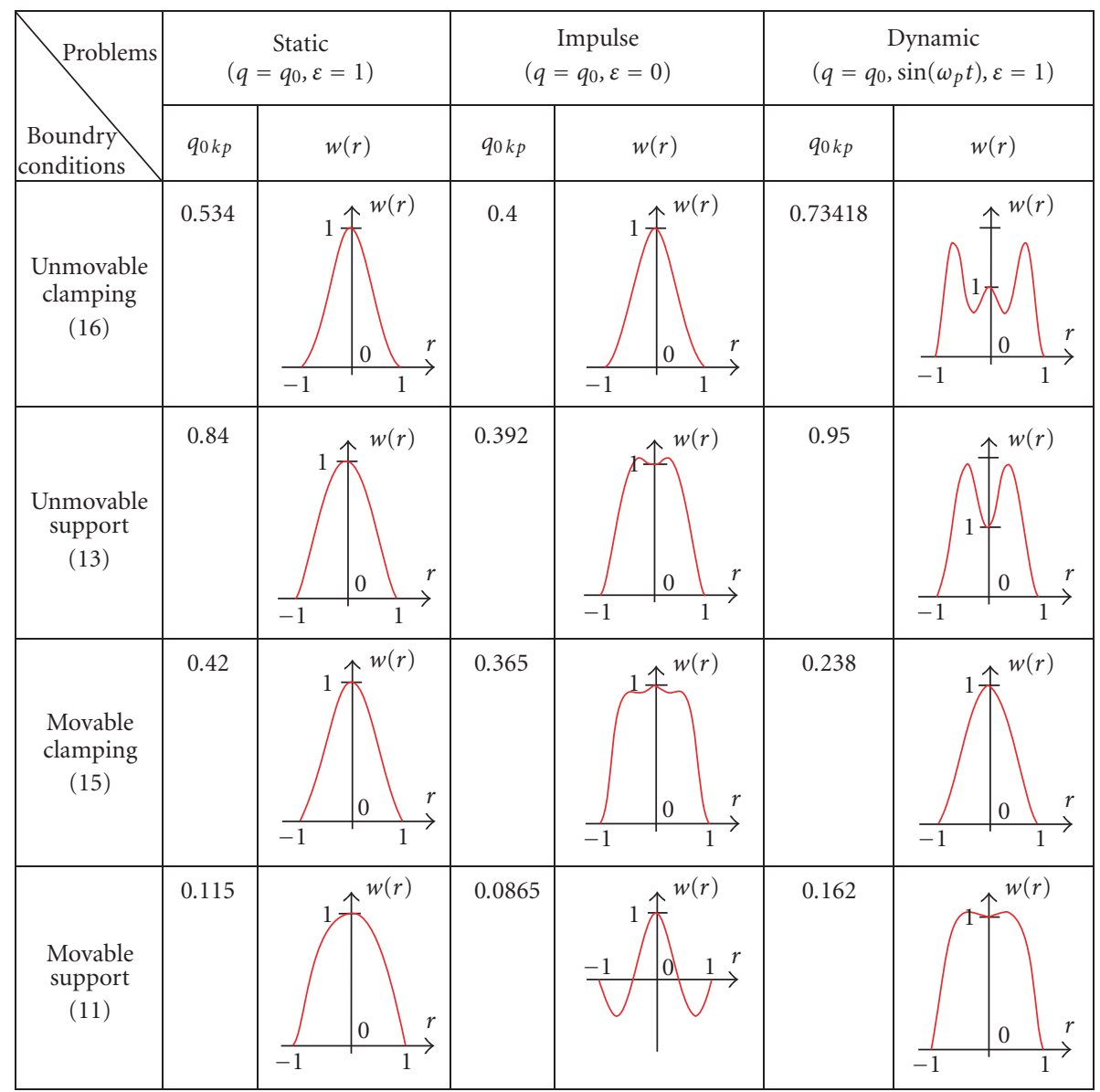

and deflection. Therefore, it is impossible to estimate either upper or lower critical loads. Contrarily, a dynamical approach proposed in this paper allows for a highly accurate prediction of critical loads, for which the so-called shell concavity and/or convexity occurs. Notice that loops occur, since the given characteristics $q_{0}(w)$ are constructed for the shell's centre, whereas other shell's points behave in independent manner, that is, the stability loss takes place not in the shell centre but in its quadrants. Therefore, the shell centre creates those loops depending on the acting load (see the graphs of deflections reported in Table 3.1). The analysis of the obtained results indicates high accuracy of our method and its high efficiency, as well as it points out the potentially wide spectrum of possible applications during solutions to various static problems.

\section{Convergence of the method with respect to spatial coordinates}

In this work, the solution to the problem for $n=20$ is studied. Initially, the problem of convergence of the solution depending on the number $n$ of shell radius partition [17] 


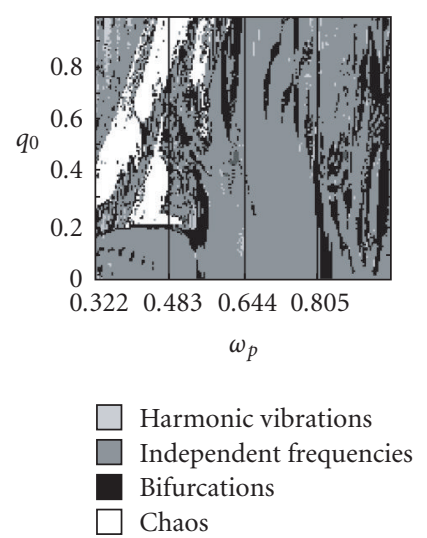

(a)

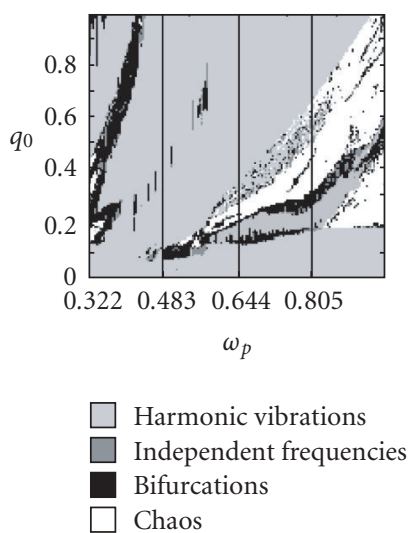

(b)

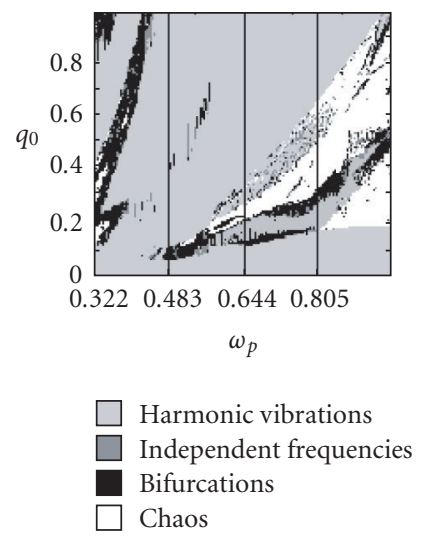

(c)

Figure 4.1. Charts of control parameters $\left\{q_{0}, \omega_{p}\right\}$.

is analysed. It has been found that $n=20$ is optimal for harmonic and chaotic areas. Power spectra for $2 n$ and $n$ of vibration character charts for control parameters $\left\{q_{0}, \omega_{p}\right\}$ coincide.

In Figure 4.1, charts serving for the identification of vibration character for control parameters $\left\{q_{0}, \omega_{p}\right\}$ with different steps of shell radius partition $n$ are reported. Namely, for Figure 4.1(a), $\left\{q_{0}, \omega_{p}\right\}$ is obtained for $n=10$; for Figure 4.1(b), the chart $\left\{q_{0}, \omega_{p}\right\}$ is obtained for $0 \leq q_{0} \leq 1$ for $n=20$; for Figure 4.1(c), the chart of control parameters is obtained for $n=30$. The comparison of charts shown in Figures 4.1(b) and 4.1(c) exhibits their coincidence, that is, the process of partition is convergent (further in this work, the notation shown in Figure 4.1 is applied).

\section{Convergence of the method with respect to the elements of the set of control parameters $\left\{q_{0}, \omega_{p}\right\}$}

Initially, the problem related to the identification of the charts of control parameters versus the step of the parameters $q_{0}$ and $\omega_{p}$ has been studied. In Figure 5.1, the charts of identification of vibration characters are analysed for three cases: (a) $100 \times 100$, (b) $200 \times 200$, and (c) $400 \times 400$. The comparison of Figures 5.1(a) and 5.1(c) exhibits their full coincidence, and hence further results have been computed with the mesh $200 \times 200$.

\section{Scenarios of the transition from harmonic to chaotic vibrations}

In what follows, we consider vibrations for a class of problems of shells theory with respect to the harmonic excitation $q=q_{0} \sin \left(\omega_{p} t\right)$ uniformly or locally distributed on the shell surface. Possible scenarios of the transition from harmonic to chaotic states are studied, and various hypotheses of the mechanism of transition from regular flow to turbulence are also illustrated and discussed. In spite of Landau's [19] hypothesis that 


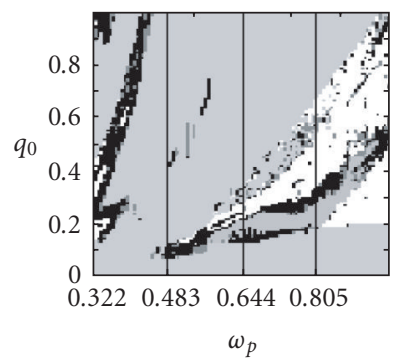

(a)

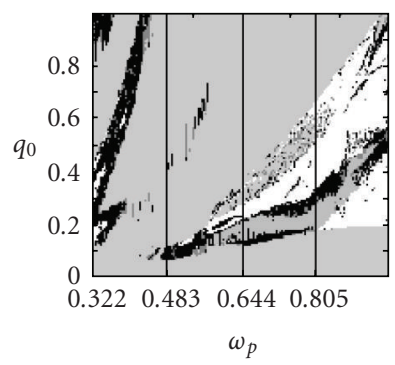

(b)

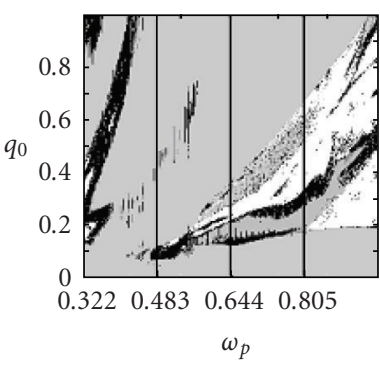

(c)

Figure 5.1. Charts of control parameters $\left\{q_{0}, \omega_{p}\right\}$.

was formulated earlier than any other, all other mechanisms are associated with finitedimensional models: Ruelle-Takens-Newhouse [24], Feigenbaum [11], and PomeauManneville [21].

It is worth noticing that so far there is no unique mechanism of transition into turbulence. In what follows, we investigate the mechanism of the occurrence of turbulence for transversal vibrations of flexible axially symmetric shells in more detail.

6.1. Feigenbaum scenario. The transition of our mechanical system from harmonic to chaotic vibrations is carried out in accordance with the Feigenbaum scenario [11]. The Feigenbaum model has been verified numerically in the case of simple mathematical models. It is well known that period-doubling bifurcations are exhibited in Rössler attractor, and others. The sequence of period doubling bifurcation is also found in our system. In Table 6.1, for central shell point with ball-type movable contour (2.11) and for $b=4$, some dependencies for boundary values of $q_{0}$ are reported, that is, phase portrait $w\left(w^{\prime}\right)$, power spectrum $S, d b\left(\omega_{p}\right)$, and Poincaré sections $w_{t}\left(w_{t+T}\right)$, where $T$ is the period of excitation. Considering the results given in Table 6.1 yields the convergent series

$$
d_{n}=\frac{q_{0, n}-q_{0, n-1}}{q_{0, n+1}-q_{0, n}}=4.65608466, \quad n=5 .
$$

Notice that theoretically predicted $d=4.66916224$. It is worth noticing that the difference between theoretical and numerical experiment is $0.28 \%$. The values of series $q_{0, n}$ and $d_{n}$ are shown in Table 6.2.

The vibrational process of axially symmetric shallow shell with a ball-type movable contour for $b=4$ exhibits chaotic attractors called Smale attractors [28]. They are also called either strange attractors or Feigenbaum-type attractors.

6.2. Ruelle-Takens-Newhouse scenario. The Ruelle-Takens-Newhouse scenario has an important scientific meaning, since it yields the example contrary to the so far existing so-called Landau-Hopf scenario [19].

In 1971, Ruelle and Takens [24] showed that a finite sequence of bifurcation is required to reach chaotic state. Initially, they found three Hopf bifurcations, and $T^{3}$ torus then created can loose its stability and chaotic attractor may occur. 
Table 6.1

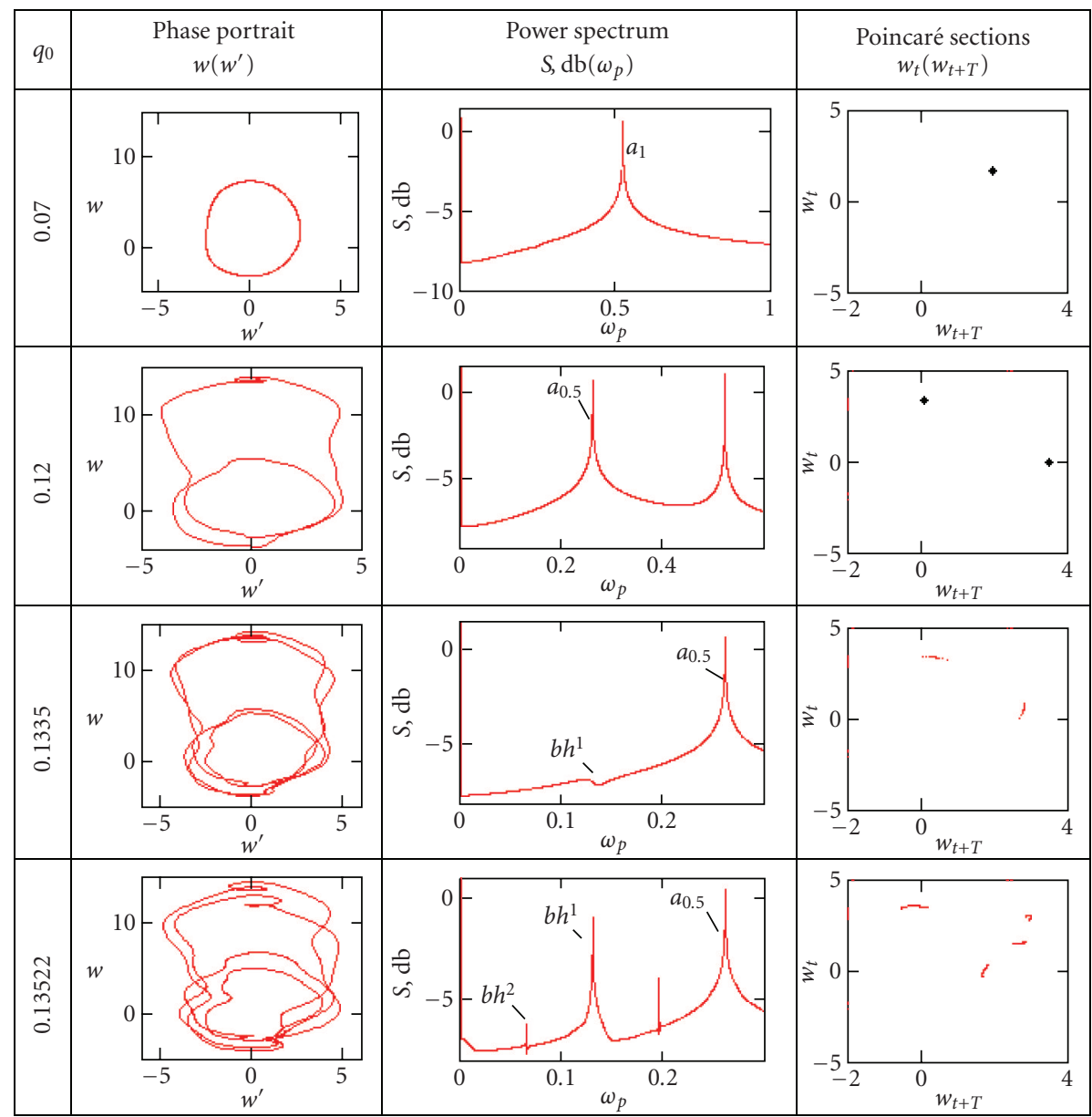

Next, in 1978, Ruelle, Takens, and Newhouse proved the theorem according to which a strange chaotic attractor is generated only through two successive Hopf bifurcations [24].

Indeed, Ruelle and Takens have shown that after Hopf bifurcations, the motion is bounded by nonsmooth manifolds with the complex topology. Such manifolds have been called strange attractors. The strange attractors do not have integer dimensions, that is, it is something between a surface and space. The fractal dimension has been studied in particular by Mandelbrot [20]. Notice that attractor obtained through the Ruelle-TakensNewhous scenario should satisfy some defined conditions, that is, if it satisfies the socalled "axiom A," then it is a chaotic one (in practice, such class of attractors rarely appears in nature). Also, a chaotic attractor is very sensitive to initial conditions.

Feigenbaum et al., and Rand et al., in 1982 independently considered the following problem: How quasiperiodic motion with two independent frequencies $\omega_{1}$ and $\omega_{2}$ on tori can be phase-locked after the substitution of small excitation. 
Table 6.1. (Continued).

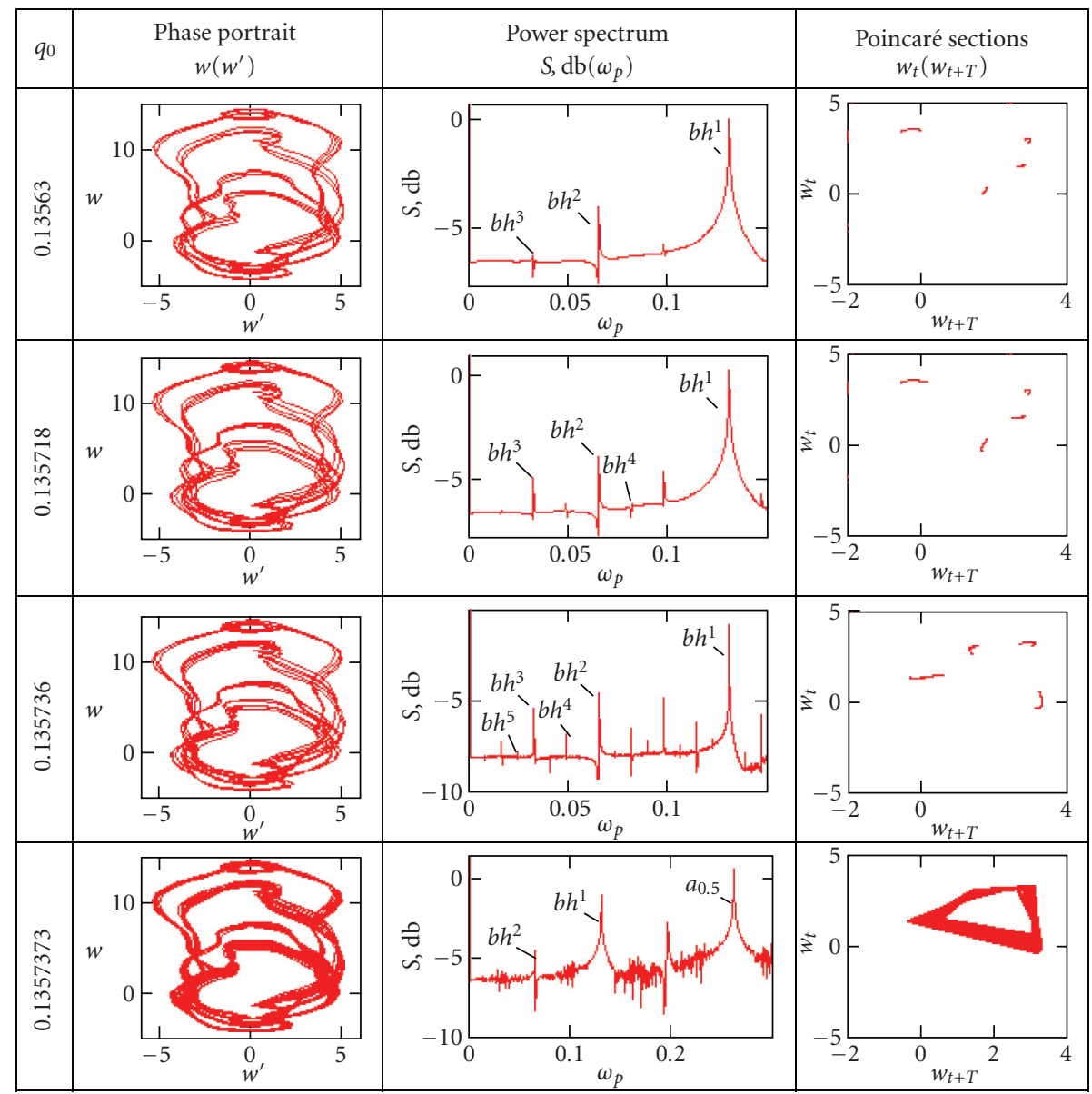

Table 6.2

\begin{tabular}{lccccc}
\hline$n$ & 1st bifurcation & 2nd bifurcation & 3rd bifurcation & 4th bifurcation & 5th bifurcation \\
\hline$q_{0, n}$ & 0.1335 & 0.13522 & 0.13563 & 0.135718 & 0.1357369 \\
$d_{n}$ & - & 4.19512 & 4.659091 & 4.656084 & - \\
\hline
\end{tabular}

Recall that due to rational values $\omega_{1} / \omega_{2}=p / q$, a trajectory closes after $q$ cycles (synchronization). However, when $\omega_{1} / \omega_{2}$ irrational, one gets the quasiperiodic motion, that is, the trajectory is never closed and occupies the whole torus surface.

6.3. Modified Ruelle-Takens-Newhouse scenario. For a spherical shell with ball-type movable face and contour (2.13), with sliding (2.15) and stiff (2.16) clamping and harmonic excitation of uniformly distributed load, the new mechanism of transition from harmonic to chaotic vibrations has been obtained. Recall that in the Ruelle-Takens- 
Table 6.3

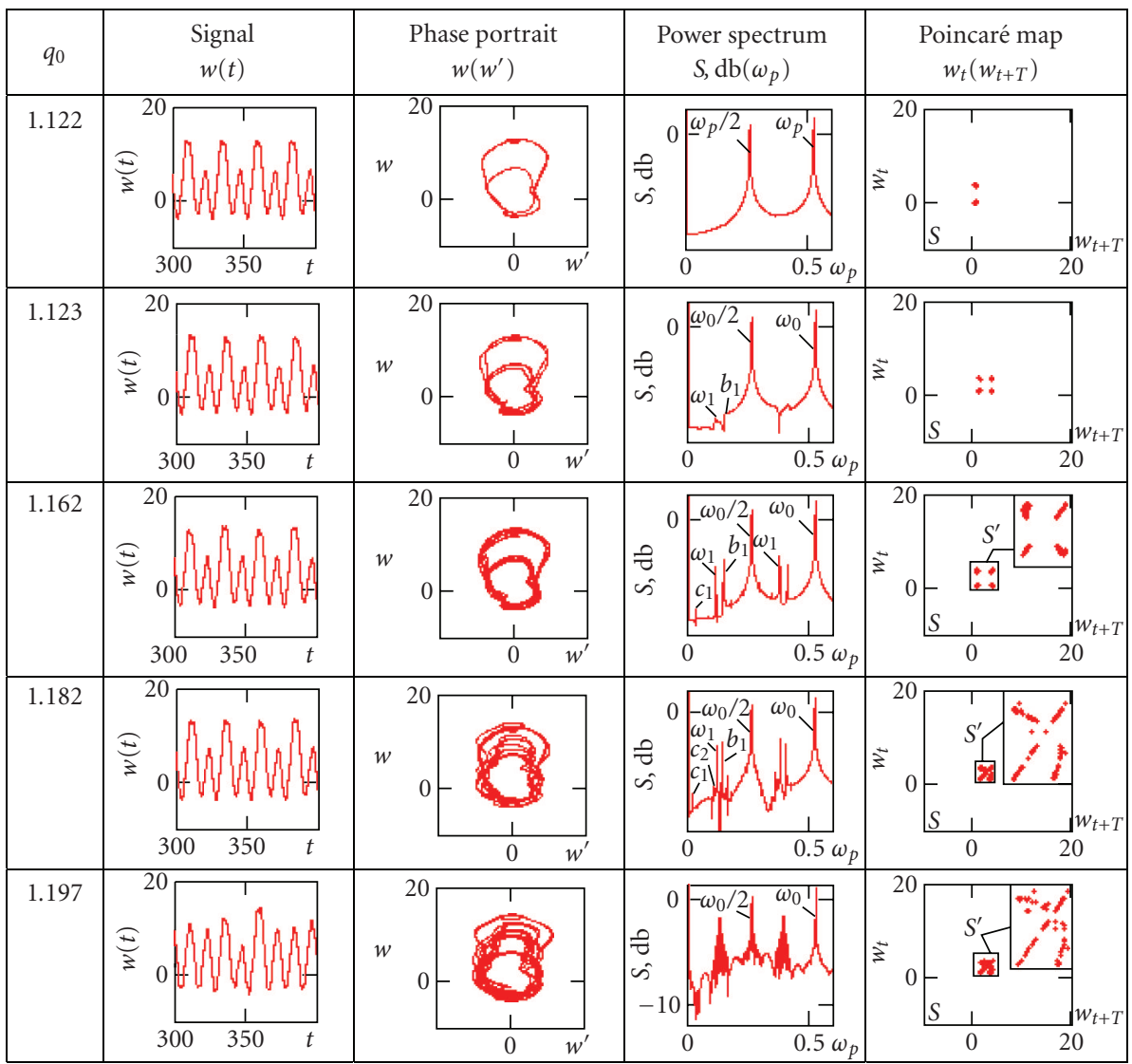

Newhouse scenario, three independent frequencies are required to reach chaos. Contrarily, in the modified scenario, the transition is realized through one independent frequency and its linear combinations with the frequency of excitation. Let us consider this novel scenario in more detail.

The applied fundamental characteristics follow: signal $w(0, t)$, phase portrait $w(\dot{w})$, power spectrum $S, d b\left(\omega_{p}\right)$, and Poincare map $w_{t}\left(w_{t+T}\right)$ in the dependency of the limiting values $q_{0}$ are given in Table 6.3. The values $q_{0}$ are called limiting because between the shown limits of $q_{0}$, the dynamical picture remains unchanged. Let us consider this scenario using example of the problem with stiff clamped resistance contour.

(1) Vibrations consist of the fundamental excitation frequency $a_{1}$, and they are harmonic. The phase portrait is composed of a limited set of one circled cycle $\left(q_{0}=\right.$ $0.68)$.

(2) Further increase of the parameter $q_{0}$ up to the value of $q_{0}=0.699$ yields a new independent frequency $b_{1}$, that is, there is two-frequency motion (with frequencies $a_{1}$ and $\left.b_{1}\right)$. Notice that the motion is not synchronized, that is, $a_{1} / b_{1}=m_{1} / n_{1}=$ 3.169 is the irrational value. 
(3) Further increase up to $q_{0}=0.7$ results in the activation of the series of linearly dependent frequencies $b_{n}=n \cdot b_{1}$ and $a_{n}=a_{1}-(n-1) b_{1}$. Due to the continuation of this process, two frequencies $a_{k}$ and $b_{k} \in\left[b_{1}, a_{1}\right]\left(q_{0}=0.706\right)$ approach each other. Then, one more series of linear combinations appears, that is, $c_{2}=b_{2}-a_{2}$, $c_{n}=x \pm c_{2}\left(x=a_{n}, b_{n}\right)\left(q_{0}=0.73418\right)$.

The increase of $q_{0}$ up to $q_{0}=0.73418$ puts the considered system into chaos. Variation of $q_{0}$ on the amount of $2 \cdot 10^{-5}$, that is, up to the value of $q_{0}=0.7342$, causes the occurrence of the shell stiff stability loss, and the system starts to vibrate again harmonically with the excitation frequency $a_{1}$. Here we may treat this process as the dynamical stability loss of spherical shells subjected to periodic harmonic excitations.

\section{The investigation of chaotic vibrations of the harmonically excited shell}

Let us consider vibrations of the harmonically excited shell. The system behaviour is investigated for three different values of the sloping parameter $b=3,4,5$, and for the following three types of clamping: ball-type movable contour (2.13), sliding clamping (2.15), and stiff clamping (2.16). In order to investigate the shell behaviour in different cases, the following characteristics are monitored: charts of control $\left\{q_{0}, \omega_{p}\right\}$ for various values of the shell sloping parameters (Figure 7.1$)$, and the graphs of the dependency $q_{0}\left(w_{\max }\right)$ (Figure 7.2).

The charts of control parameters $\left\{q_{0}, \omega_{p}\right\}$ for all the problems associated with uniform harmonic excitations have been constructed (see Figure 7.1). Three vertical lines correspond to the following frequencies: the middle line corresponds to $\omega_{p}=\omega_{0}$ (frequency of linear vibrations), whereas the left and right lines correspond to $\omega_{p}-\omega_{p} / 4$ and $\omega_{p}+\omega_{p} / 4$, respectively. Notice that the analysis of three charts exhibits all possible manifolds of complex vibrations of spherical shells.

According to the investigation of the shell behaviour in various conditions, the increase of the sloping parameter $b=3,4,5$ yields the increase of chaotic zone for any type of clamping (see the charts of control parameters). However, the increase of the sloping parameter does not change the scenario of transition from harmonic to chaotic vibrations. Comparing the shell behaviour depending on the clamping type, the following observations are made. Zones of chaotic vibrations are larger for a ball-type unmovable contour and stiff clamping of the shell, and in the majority of the analysed cases, a route to chaos is realized through the modified Ruelle-Takens-Newhouse scenario [24], proposed in this work. In the case of the ball-type movable contour (2.11) and movable clamping (2.16), the Feigenbaum scenario [11] dominates.

For all types of clamping and the shell sloping parameter values, the dependencies $q_{0}\left(w_{\max }\right)$ (Figure 7.2$)$ are constructed. Under the obtained graphs, the scales characterizing various signal types are shown.

In Figure $7.2(\mathrm{a})$, in the graph of dependency $q_{0}\left(w_{\max }\right)$ (for the shell with the ball-type movable contour and with sloping parameter $b=3$ ), one inflection point as well as firstorder discontinuity are observed. In these points, the change of signal types occurs, which is manifested by the change of color in the scale of vibrations type. In Figure 7.2(b), the curve associated with the same type of clamping and with sloping parameter $b=4$ is reported. Observe the inflection point and the series of stiff bifurcations. In addition, the 


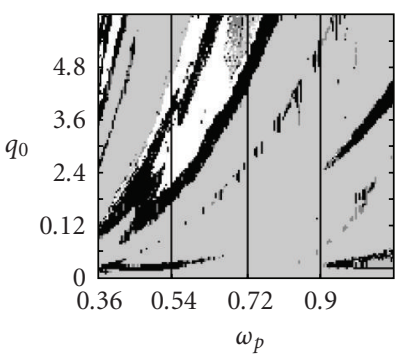

(a) $b=3$.

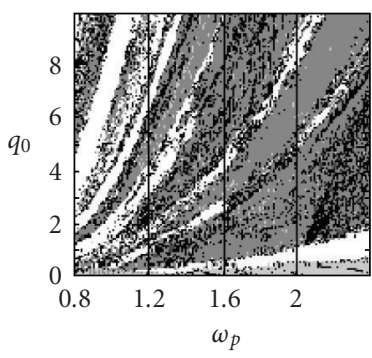

(d) $b=3$.

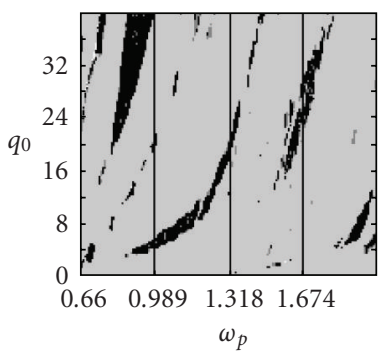

(g) $b=3$.

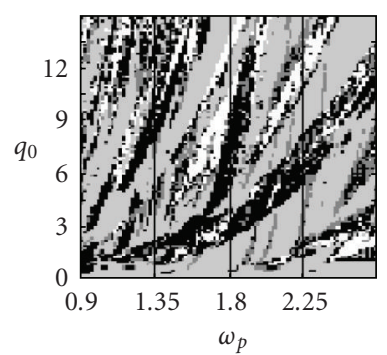

(j) $b=3$.

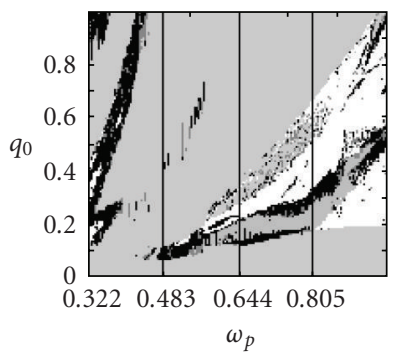

(b) $b=4$.

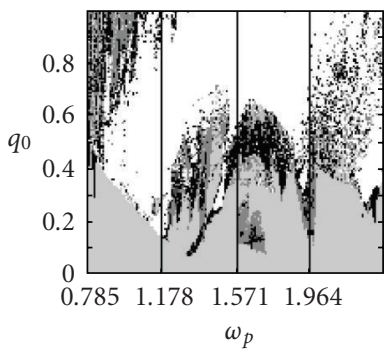

(e) $b=4$.

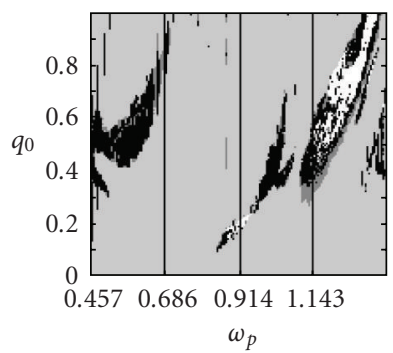

(h) $b=4$.

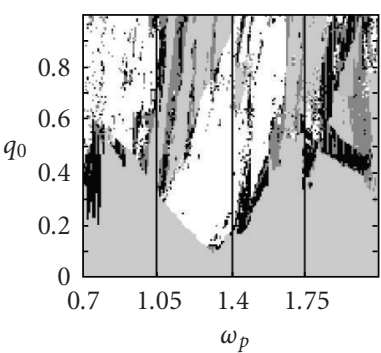

(k) $b=4$.

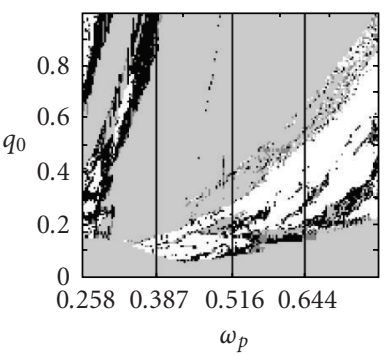

(c) $b=5$.

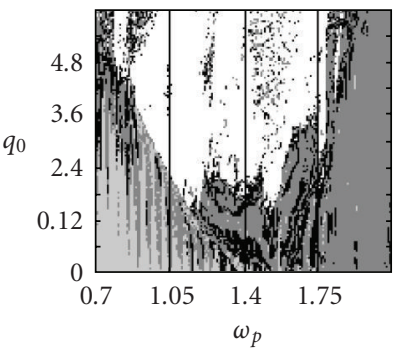

(f) $b=5$.

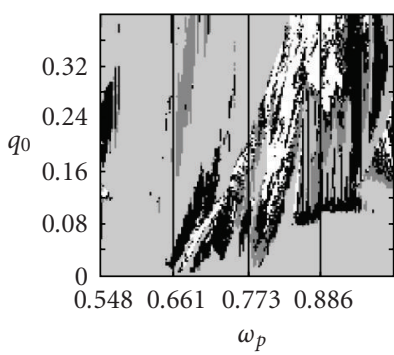

(i) $b=5$.

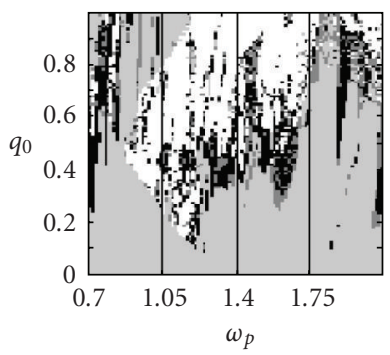

(l) $b=5$.

Figure 7.1. Charts of control parameters $\left\{q_{0}, \omega_{p}\right\}$. (a)-(c) Ball-type movable contour. (d)-(f) Balltype unmovable contour. (g)-(i) Sliding clamping. (j)-(l) Stiff clamping. 


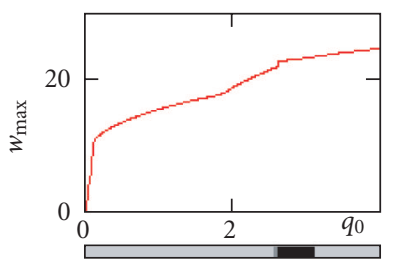

(a) $b=3$.

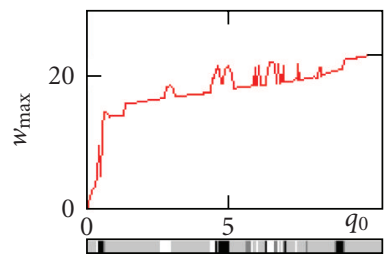

(d) $b=3$.

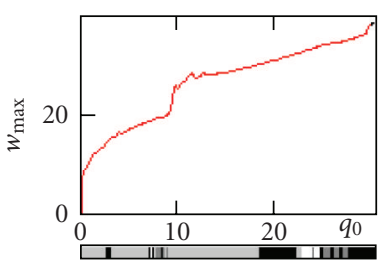

(g) $b=3$.

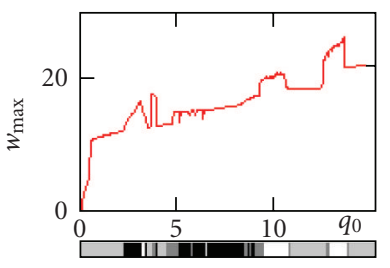

(j) $b=3$.

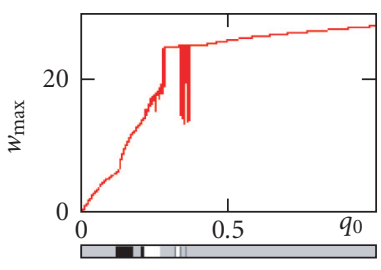

(b) $b=4$.

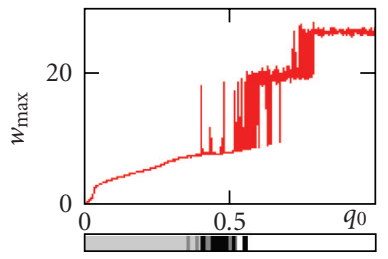

(e) $b=4$.

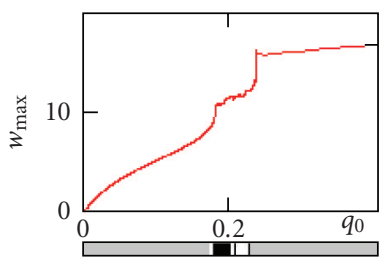

(h) $b=4$.

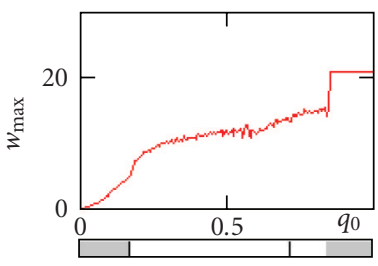

(k) $b=4$.

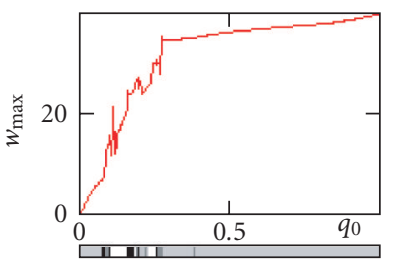

(c) $b=5$.

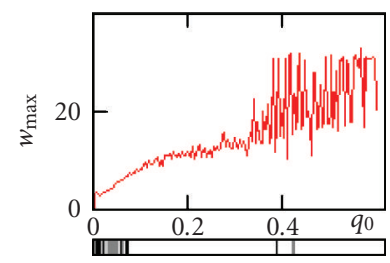

(f) $b=5$.

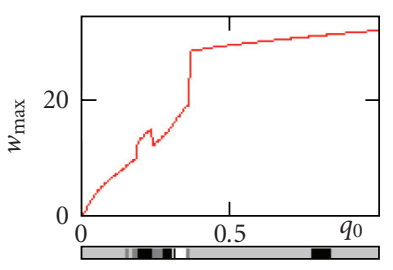

(i) $b=5$.

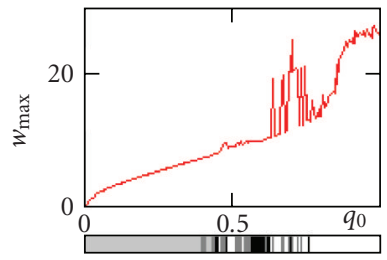

(1) $b=5$.

Figure 7.2. Dependencies $q_{0}\left(w_{\max }\right)$. (a)-(c) Ball-type movable resistance contour. (d)-(f) Ball-type unmovable resistance contour. (g)-(i) Sliding clamping. (j)-(l) Stiff clamping.

change of vibrations type is manifested by colors associated with signal types. Owing to the increase of $b$ parameter, chaotic vibrations are observed for the smaller amplitude of the existing load than those for $b=3$. The further increase of the sloping parameter up to $b=5$ (Figure 7.2(c)) yields the increase of the first-order discontinuities, and of the surface of chaotic zones. The analogical picture is produced also for other boundary conditions. 


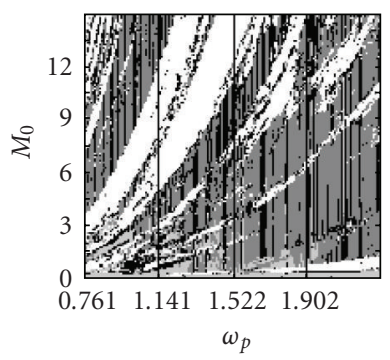

(a)

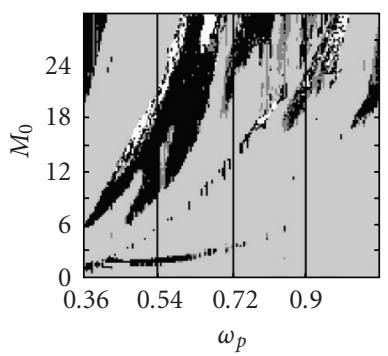

(d)

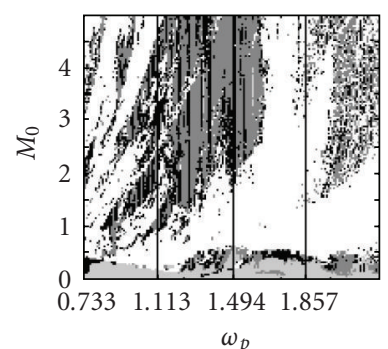

(b)

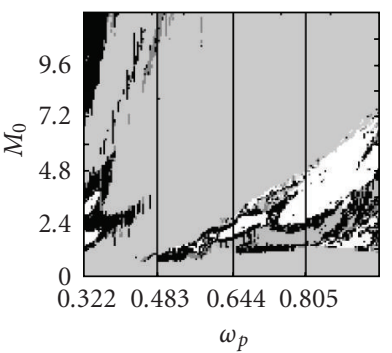

(e)

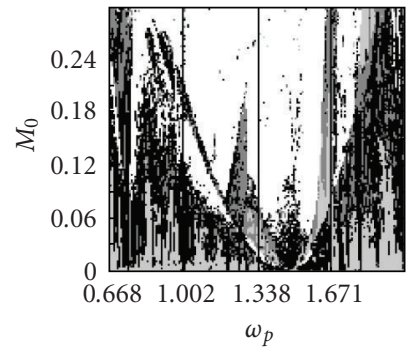

(c)

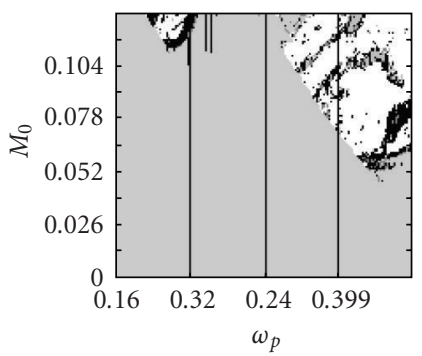

(f)

Figure 8.1. Charts of control parameters $\left\{M_{0}, \omega_{p}\right\}$.

\section{Complex vibrations of a shell subjected to harmonic moment}

In this section, we consider vibrations of the outlined class problems of theory of shells in the case of harmonic resistance moment excitation $M=M_{0} \sin \left(\omega_{p} t\right)$. This moment drives both contours of the shell with ball-type support (2.12) for $b=3, b=4, b=$ 5 , and with unmovable contour (2.14) for $b=3, b=4, b=5$ for $q=0$ in (2.1) and (2.10).

For all the considered problems, charts of control parameters $\left\{M_{0}, \omega_{p}\right\}$ are constructed. For ball-type unmovable contour with $b=3, \varepsilon=0.1$, see Figure 8.1(a), for $b=4$, $\varepsilon=0.1$, see Figure 8.1(b), and for $b=5, \varepsilon=0.1$, see Figure 8.1(c), as well as for ball-type movable contour $b=3, \varepsilon=0.1$, see Figure 8.1(d), for $b=5, \varepsilon=0.1$, see Figure 8.1(e), for $b=5, \varepsilon=0.1$, see Figure 8.1(f).

The analysis of the dependencies $\left\{M_{0}, \omega_{p}\right\}$ for these types of problems implies that in the case of the shell with ball-type unmovable contour subjected to harmonic moment (Figures 8.1(a), 8.1(b), and 8.1(c)), a transition from harmonic to chaotic vibrations is realized either according to the Feigenbaum [11] or to the modified Ruelle-TakensNewhouse [24] scenarios. In the problem with ball-type movable contour (Figures 8.1(d), 8.1(e), and 8.1(f)), the system is transited into chaos only according to the Feigenbaum scenario. The Feigenbaum model is well verified via numerical experiment applied to simple mathematical models. The same behaviour is also detected in the case of our 
Table 8.1

\begin{tabular}{lcccc}
\hline$N$ & 1st bifurcation & 2nd bifurcation & 3rd bifurcation & 4th bifurcation \\
\hline$q_{0, n}$ & 0137 & 0.1395 & 0.14013 & 0.1402667 \\
$d_{n}$ & - & 3.968253968 & 4.60863204 & - \\
\hline
\end{tabular}

analysed shell with a ball-type movable contour and subjected to harmonic moment. The following Feigenbaum constant is computed for $b=4$ :

$$
d_{n}=\frac{q_{0, n}-q_{0, n-1}}{q_{0, n+1}-q_{0, n}}=4.60863204, \quad n=4(\text { see Table } 8.1) .
$$

Notice that the theoretical value $d=4.66916224$, and hence the difference between theoretical and numerical results is $1.289 \%$.

In two charts of control parameters $\left\{M_{0}, \omega_{p}\right\}$, constructed for the shell with a ball-type unmovable contour, large zones of chaotic behaviour are visible. Contrarily, for a balltype movable contour, in the chart of control parameters $\left\{M_{0}, \omega_{p}\right\}$, significantly fewer zones with chaotic vibrations appear. It is worth noticing that during the increase of the shell rise, chaotic zones essentially increase.

It is clear that the system dynamics essentially depends on loading type for the same boundary and initial conditions, and the shell geometry.

For all problems, the graphs of the dependencies $w_{\max }\left(M_{0}\right), F_{\max }\left(M_{0}\right), M_{1 \max }\left(M_{0}\right)$, where $M_{1}=-\left(\partial^{2} w / \partial r^{2}\right)-v(\partial w / r \partial r)$ (for $\left.r=\Delta\right)$, are constructed. For a ball-type movable contour with $b=3, \varepsilon=0.1$, see Figure 8.2(a), for a ball-type unmovable contour $b=4, \varepsilon=0.1$, see Figure 8.2(b), for $b=5, \varepsilon=0.1$, see Figure 8.2(c), for a ball-type unmovable contour $b=3, \varepsilon=0.1$, see Figure 8.2(d), for $b=4, \varepsilon=0.1$, see Figure 8.2(e), and for $b=5, \varepsilon=0.1$, see Figure 8.2(f).

During investigations of the shell vibrations, its stress-strain state plays the most important role, and hence its dependence on the amplitude $M_{0}$ is studied. One of the key problems is associated with the consideration of the deflection function and stresses versus parameter $M_{0}$ variations. As it is well known in the theory of flexible shells, the full shell stress consists of the stresses in the mean shell surface and of the bending stresses. The stress function $F$ plays an important role in the stress determination in the shell mean surface, and in the case of bending stresses-it is the moment $M_{1}$. Let us construct the characteristics $w_{\max }\left(M_{0}\right), F_{\max }\left(M_{0}\right), M_{1 \max }\left(M_{0}\right)$. The analysis of the given characteristics indicates that all of them describe in the same manner the occurrence of dynamical stability loss via stiff bifurcation. It is evidently shown in the characteristic $M_{1 \text { max }}\left(M_{0}\right)$, since it contains the second derivative of the deflection function with respect to a coordinate. Dependencies $w_{\max }\left(M_{0}\right), F_{\max }\left(M_{0}\right), M_{1 \max }\left(M_{0}\right)$, the bifurcation scale, and the Lyapunov exponents well coincide [7]. The analysis of the mentioned characteristics yields the conclusion that the occurrence of timing chaos implies the occurrence of spatial chaos, that is, timing and spatial chaos are mutually coupled. It is worth noticing that chaos of shell's stress-strain state is also observed. 


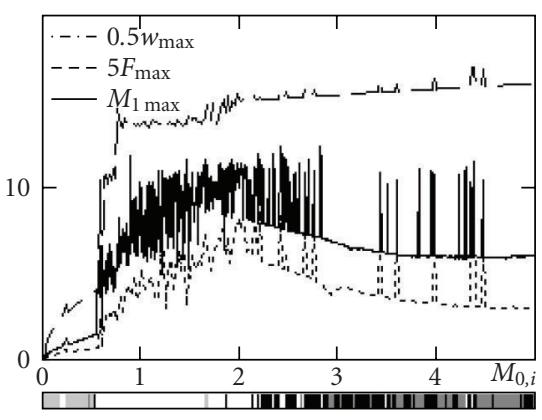

(a)

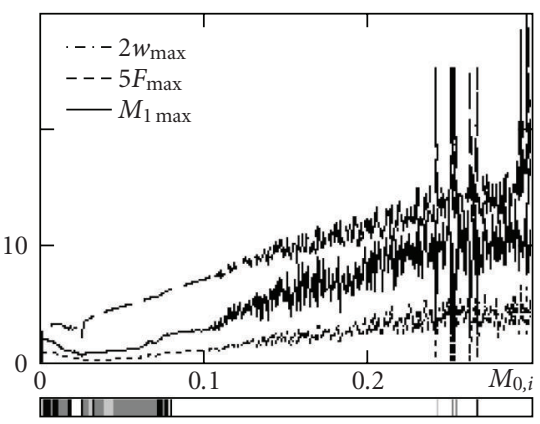

(c)

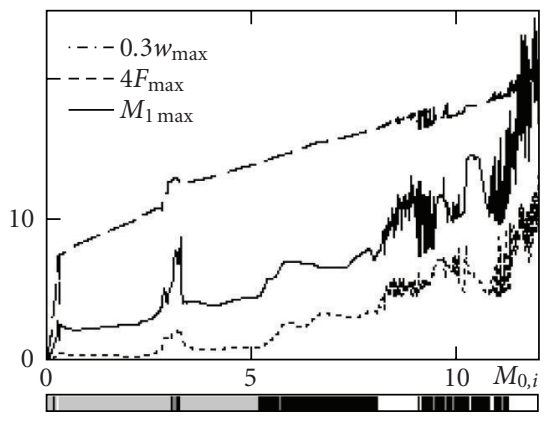

(e)

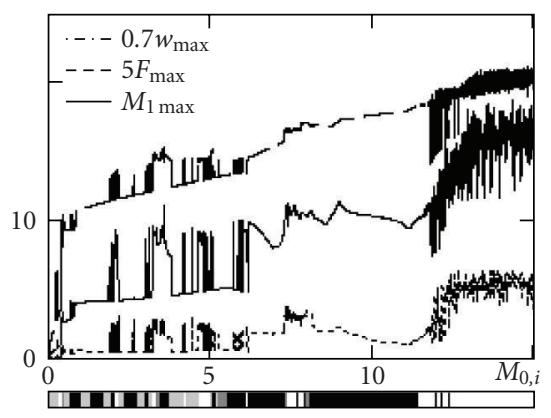

(b)

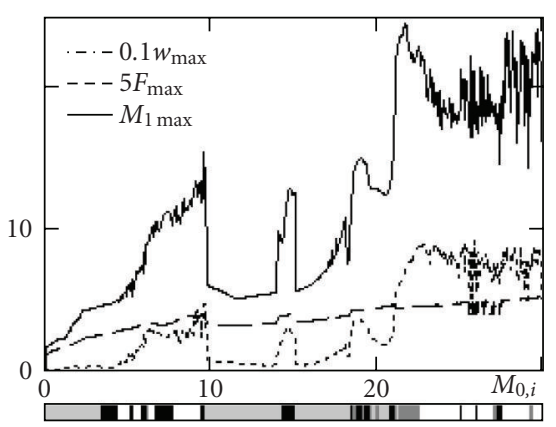

(d)

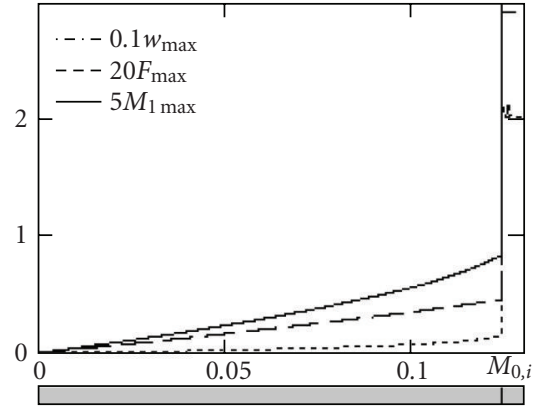

(f)

Figure 8.2. Dependencies $w_{\max }\left(M_{0}\right), F_{\max }\left(M_{0}\right), M_{1 \max }\left(M_{0}\right)$.

\section{Behaviour of shells subjected to a local harmonic load}

Consider the shell with a ball-type contour subjected to two types of local loads: (i) $q=q_{0} \sin \left(\omega_{p} t\right)$ is loaded in five points $8 \leq i \leq 12$, when $0 \leq i \leq n, i, n \in N$, in the neighborhood of the fourth one (in other points $q=0$ ); (ii) load $q=q_{0} \sin \left(\omega_{p} t\right)$ is applied to five points $0 \leq i \leq 4$, where $0 \leq i \leq n, i, n \in N$, in the neighborhood of the centre (in other points $q=0$ ). 


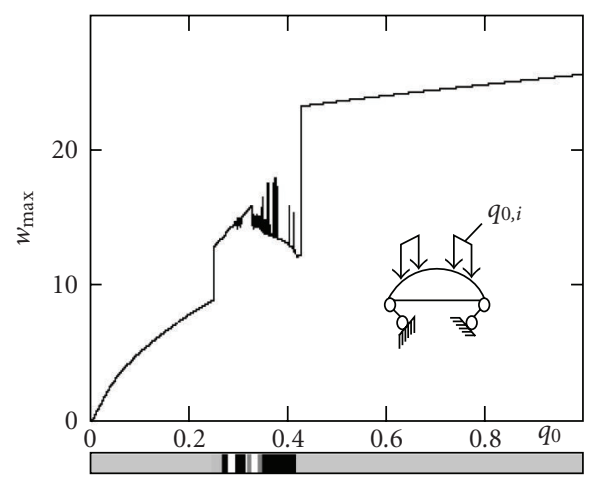

(a)

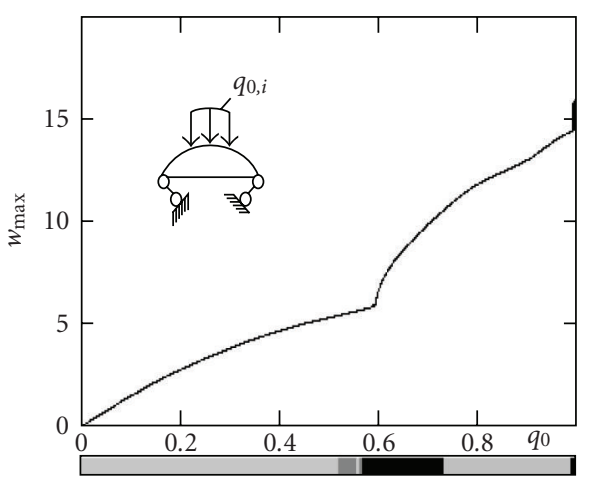

(b)

Figure 9.1. Dependency $w_{\max }\left(q_{0}\right)$.

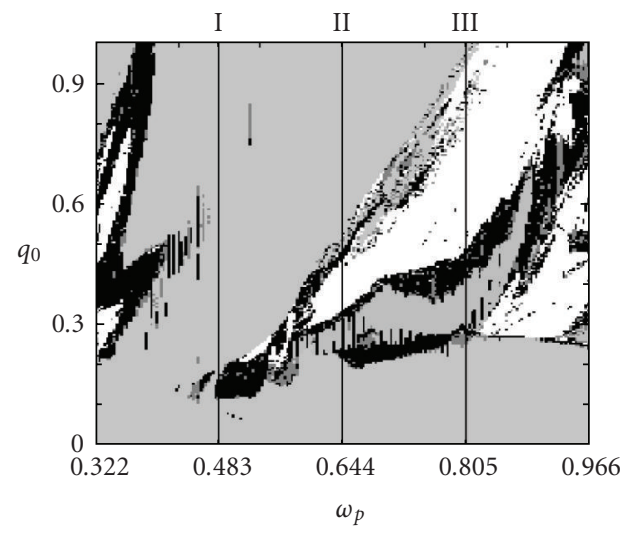

(a)

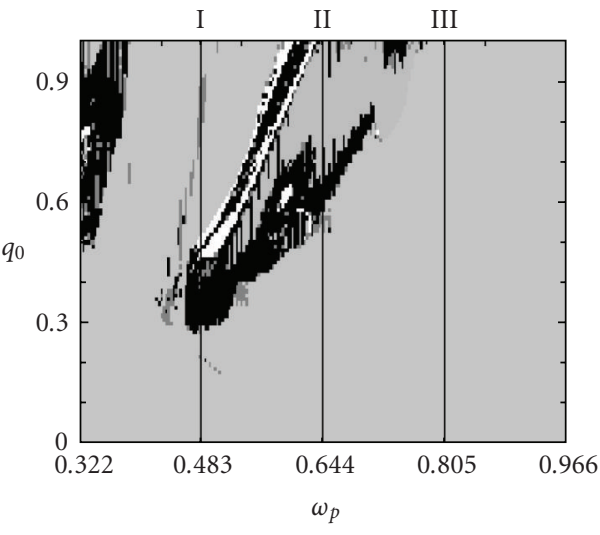

(b)

Figure 9.2. Chart of control parameters $\left\{q_{0}, \omega_{p}\right\}$.

The graphs of the dependency $w_{\max }\left(q_{0}\right)$ are given for the first type in Figure 9.1(a), whereas for the second type-in Figure 9.1(b). In the case of first shell loading type, two stiff bifurcations appear. The first stability loss occurs during the transition from harmonic vibrations to first Hopf bifurcation, whereas the second one is associated with the transition from chaotic to harmonic vibrations. The signal-type scale exhibits the existence of the Feigenbaum scenario. The dependency $w_{\max }\left(q_{0}\right)$ is more smoothened; there is no first-order discontinuity, as in the first case. In the scale of signal type, a small zone of soft bifurcations appears, and there are no chaotic zones.

Numerical experiment yields five Hopf bifurcations, and the obtained Feigenbaum constant is equal to 4.67784 (the difference in comparison to the theoretical value is $0.168 \%)$. 
Table 10.1

\begin{tabular}{cccc}
\hline Problem & Distributed load & Local load & Resistance moment \\
\hline 1 & $q=q_{0} \cdot \sin \left(\omega_{p} \cdot t\right)$ & - & - \\
2 & - & $q=q_{0} \cdot \sin \left(\omega_{p} \cdot t\right)$ & - \\
3 & - & - & $M=M_{0} \cdot \sin \left(\omega_{p} \cdot t\right)$ \\
4 & $q=q_{0} \cdot \sin \left(\omega_{p} \cdot t\right)$ & $q_{1}=0.6 \cdot \sin (0.725 \cdot t)$ & - \\
5 & $q=q_{0} \cdot \sin \left(\omega_{p} \cdot t\right)$ & - & $M_{1}=9.6 \cdot \sin (0.886 \cdot t)$ \\
\hline
\end{tabular}

For both types of local shell loading, the charts of control parameters $\left\{q_{0}, \omega_{p}\right\}$ are constructed (first loading type is given in Figure 9.2(a), second loading type-Figure 9.2(b)). In the chart of control parameters $\left\{q_{0}, \omega_{p}\right\}$ and for the first loading type, one may observe a large zone of chaotic vibrations with high frequencies. Small zones of Hopf bifurcations associated with low frequencies and "drops" of independent frequencies as well as their linear combinations are also visible. Vibration character is different now in comparison to the first loading type. In the chart of control parameters, large zones of regular vibrations are visible. The small zone of chaotic vibrations is shifted into the area of low frequencies. "Drops" of independent frequencies and their linear combinations do not appear.

\section{Control of chaotic vibrations of flexible spherical shells}

It is well known that a chaotic attractor of a dynamical system consists of the countable set of saddle cycles with different periods and that its representing point (trajectory) visits close neigbourhood of each of them. If in this time instant one tries to stabilize a saddle cycle, then trajectory will remain in its vicinity, and the system exhibits periodic motions. On the other hand, problems of the control of chaos in interacting systems are indirectly associated with problems of synchronization. In what follows, by applying some targetoriented excitations, the defined chaotic subsets corresponding to synchronized motions of identical systems can be transformed to the stable ones with respect to some chosen directions, keeping the rest of them as unstable ones in other directions. One may expect the controlled transition from unsynchronized chaotic vibrations to regime of fully synchronized chaos as a result. In this work, the mentioned control is realized with the help of target-oriented shell excitation. The shell is sinusoidally excited $q=q_{0} \sin \left(\omega_{p} t\right)$ and two types of periodic excitations are applied:

(i) additional local transversal harmonic load is applied in five points $8 \leq i \leq 12$, where $0 \leq i \leq n, i, n \in Z$;

(ii) additional resistant harmonic moment.

The mentioned excitations are of two types: with a fixed frequency and with synchronized frequencies.

In order to study the behaviour of the shell subjected to two exciting loads with different frequencies (problems 3-5, Table 10.1), the mathematical model of two-frequency vibrating system is realized and charts of vibration character for control parameters $\left\{q_{0}, \omega_{p}\right\}$ are constructed (see Figure 10.1). 


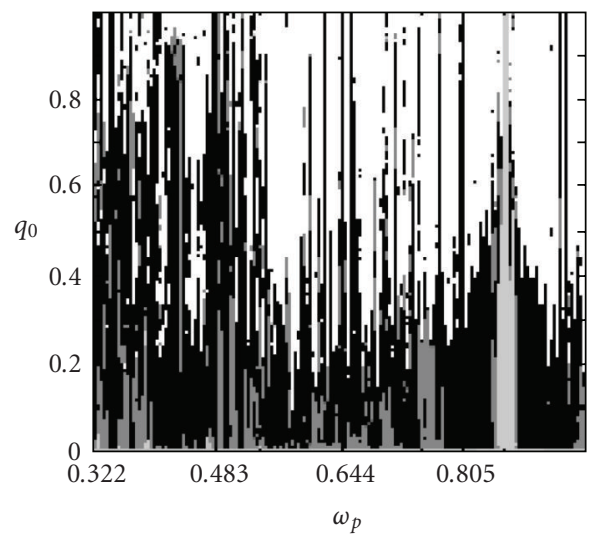

(a)

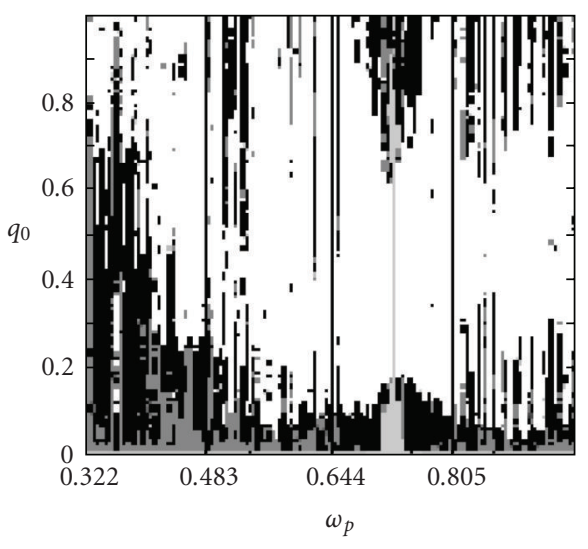

(b)

Figure 10.1. Charts of different vibrations in the plane of $\left\{q_{0}, \omega_{p}\right\}$.

In all charts, large zones of chaotic vibrations are remarkable. The increase of the frequency of local excitation causes the expansion of chaotic zones.

The system is transited into harmonic vibrations only in the case when frequencies of two exciting forces are close to each other.

In this case, the area of harmonic vibrations (located in vicinity of the frequency of either exciting force or exciting resistance moment (Figure 10.1(b))) on the charts of vibration characters is studied in more detail, with the use of the dependencies $w_{\max }\left(q_{0}\right)$. Here, five loading types are reported. Notice that the number of curves shown in Figure 10.2 and the number of problems reported in Table 10.1 are identical.

In Figures 10.2(a) and 10.2(b), the graphs related to the problems 1, 2, $4(1,3,5)$ are constructed. In curves 1, 2, 3 (Figures 10.2(a) and 10.2(b)), the first-order discontinuities are shown, indicating the existence of stiff stability loss. The occurrence of stiff stability loss is manifested also through signal-type scales, where in the corresponding time instants, a change of vibrations appears. In the scales of signal types $1,2,3$, one may observe a few zones of chaotic vibrations with independent frequencies and bifurcations. In both problems, the synchronization of two frequencies of exciting loads deletes the zone of chaotic vibrations, bifurcation and that of independent frequencies. In the scales of signal types 4 (Figure 10.2(a)) and 5 (Figure 10.2(b)), only harmonic vibrations are visible. Dependencies $w_{\max }\left(q_{0}\right) 4$ (Figure 10.2(a)) and 5 (Figure 10.2(b)) are smooth, and first-order discontinuities do not appear.

Notice that the values of the frequency $\omega_{p}$ and moment $M_{0}$ or $\omega_{p}$ and the amplitude of the exciting force have been chosen according to the experiments carried out. It has been detected that the increase of the added excitation causes the expansion of a zone of harmonic vibrations.

The analysis of the control parameter charts of the problems 4 and 5 (Table 10.1) shows that in the case of frequencies without local and distributed loads or a resistance moment and distributed load $\omega_{p}=0.859$, the vertical zone occurs in the charts of control 


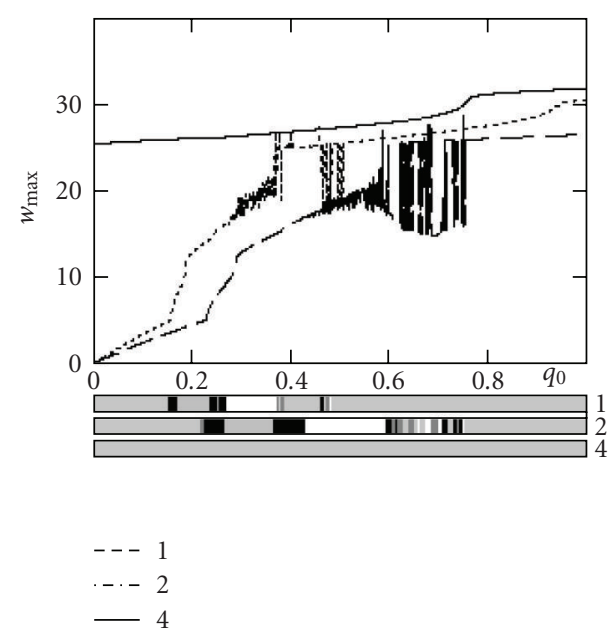

(a)
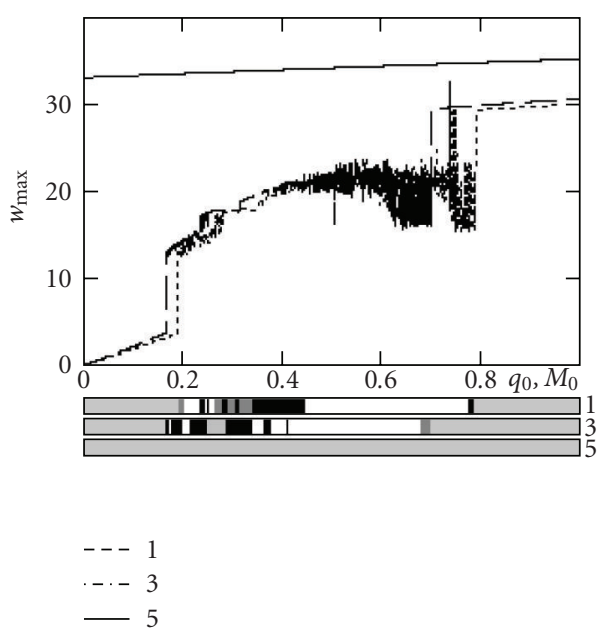
)
(b)

Figure 10.2. Dependencies $w_{\max }\left(q_{0}\right)$.

parameter $s$, whereas for all $q_{0} \in[0,1]$ harmonic vibrations are visible. Therefore, it is required to consider the problem when the shell is subjected to a uniformly distributed load and local load action, or to harmonic resistance moment with synchronized frequencies in both excitations (Table 10.2).

In Figure 10.3, the charts of vibration character for the control parameters $\left\{q_{0}, \omega_{p}\right\}$, $\left\{M_{0}, \omega_{p}\right\}$ are reported. The first three charts have been already displayed in Figures 7.1(b), 8.1(e), and 9.2(a). They are repeated here to get more deep insight of the problem. The analysis of the obtained results yields the observation that a forced synchronization of external excitations shifts the analysed mechanical system into another type of vibrations. It is worth noticing that chaotic zones are almost fully cancelled. Recall that they appeared previously in the charts $\left\{q_{0}, \omega_{p}\right\},\left\{M_{0}, \omega_{p}\right\}$ (Figures 10.3(a), 10.3(b), 10.3(c), problems 1, 2, 3; Table 10.2) practically in the same places. In the case of fourth loading type (Figure 10.3(d), problem 4; Table 10.2), the exhibited vibrations are mostly harmonic. Only a small zone of chaos and bifurcations with low and high frequencies remain. In the case of fifth type of loading (Figure 10.3(e), problem 5; Table 10.2), there are bifurcation zones, and small zones of chaos only associated with low frequencies are exhibited.

Similar considerations have been carried out also for the shell with a ball-type unmovable resistance contour and a sloping parameter $b=4$. The results of these considerations are reported in Figure 10.4.

According to earlier obtained results concerning a ball-type moving resistance contour, only some problems of chaos control are addressed. In other words, the methods of chaos control used efficiently before are applied now to the shell with a ball-type unmovable resistance contour. Although charts of control parameters corresponding to the uniformly distributed harmonic load (Figure 10.4(a)) and to harmonic resistance moment 
Table 10.2

\begin{tabular}{cccc}
\hline Problem & Distributed load & Local load & Resistance moment \\
\hline 1 & $q=q_{0} \cdot \sin \left(\omega_{p} \cdot t\right)$ & - & - \\
2 & - & $q=q_{0} \cdot \sin \left(\omega_{p} \cdot t\right)$ & - \\
3 & - & - & $M=M_{0} \cdot \sin \left(\omega_{p} \cdot t\right)$ \\
4 & $q=q_{0} \cdot \sin \left(\omega_{p} \cdot t\right)$ & $q_{1}=0.6 \cdot \sin \left(\omega_{p} \cdot t\right)$ & - \\
5 & $q=q_{0} \cdot \sin \left(\omega_{p} \cdot t\right)$ & - & $M_{1}=9.6 \cdot \sin \left(\omega_{p} \cdot t\right)$ \\
\hline
\end{tabular}

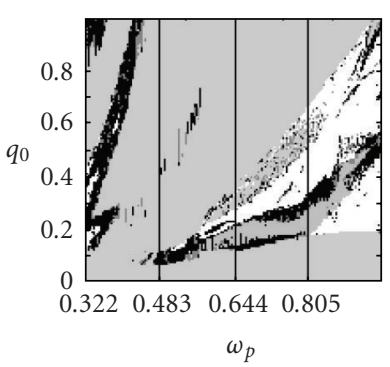

(a)

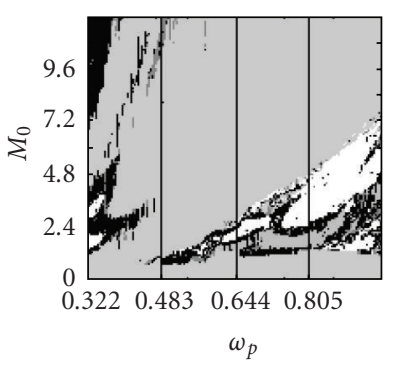

(b)

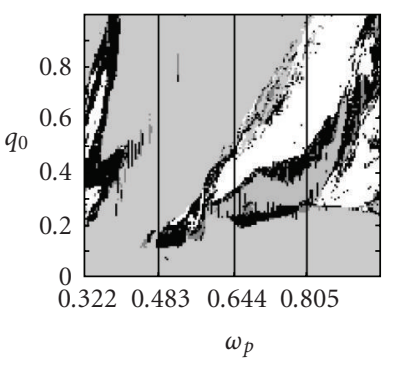

(c)

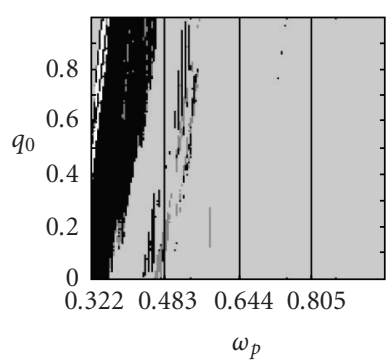

(d)

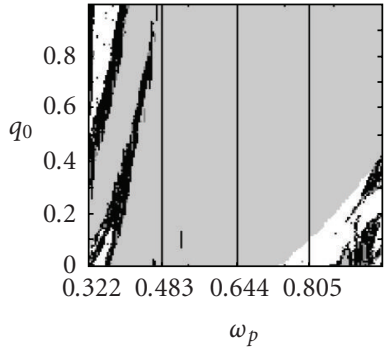

(e)

Figure 10.3. Charts of types of vibrations.

(Figure 10.4(b)) have been earlier constructed, they are repeated for the sake of clarity. Besides, charts describing behaviour of the shell subjected to a distributed load and harmonic moment, as well as to local and continuous loading, are constructed. All applied loads are shown in Table 10.3. The phase shift of the local load on amount of $\pi$ means that the local load works in antiphase with respect to a continuous load. Interaction of both added loads yields the decrease of chaotic vibrations and increase of harmonic vibrations. Notice that the action of the added local load is more efficient from the point of view of energy loss, as well as from the point of view of chaos control. The key result obtained in the course of our study is the following. The methods of control of chaotic vibrations of elastic spherical hells are the same for both ball-type movable and unmovable clampings. 


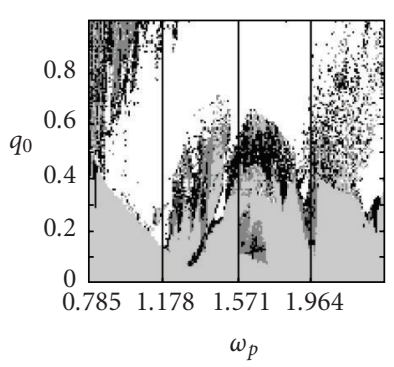

(a)

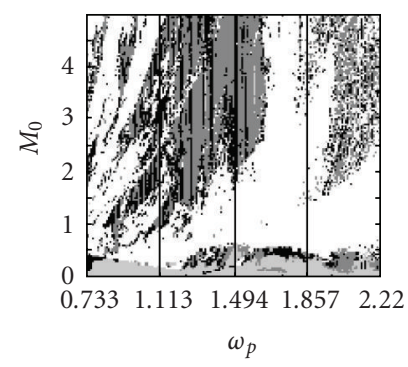

(b)

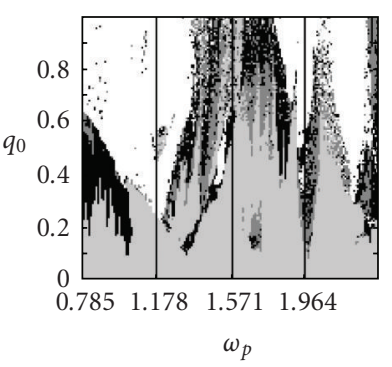

(c)

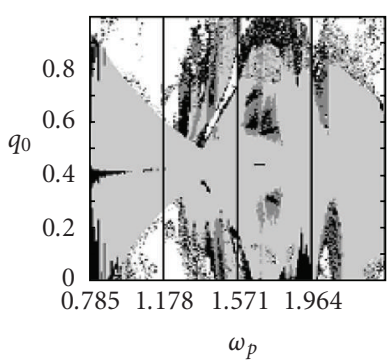

(d)

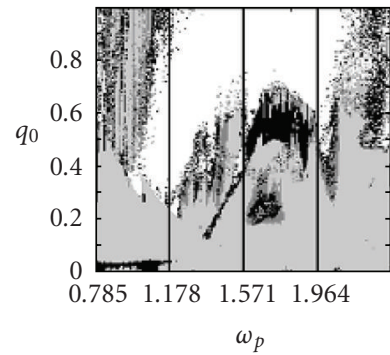

(e)

Figure 10.4. Charts of various vibrations in the planes $\left\{q_{0}, \omega_{p}\right\},\left\{M_{0}, \omega_{p}\right\}$.

Table 10.3

\begin{tabular}{cccc}
\hline Problem & Distributed load & Local load & Resistance moment \\
\hline 1 & $q=q_{0} \cdot \sin \left(\omega_{p} \cdot t\right)$ & - & - \\
2 & - & $q=q_{0} \cdot \sin \left(\omega_{p} \cdot t\right)$ & - \\
3 & - & - & $M=M_{0} \cdot \sin \left(\omega_{p} \cdot t\right)$ \\
4 & $q=q_{0} \cdot \sin \left(\omega_{p} \cdot t\right)$ & $q_{1}=\sin \left(\omega_{p} \cdot t-\pi\right)$ & - \\
5 & $q=q_{0} \cdot \sin \left(\omega_{p} \cdot t\right)$ & - & $M_{1}=0.5 \cdot \sin \left(\omega_{p} \cdot t\right)$ \\
\hline
\end{tabular}

\section{Conclusions}

A brief conclusion follow. Control of chaos is understood as a transformation of chaotic system behaviour into regular or chaotic one but with other properties resulting from target-oriented excitations applied to our shell, that is, harmonic resistance moment or harmonic local load with synchronized frequencies (the so-called forced synchronization of different exciting frequencies).

Therefore, by variation of the load, one may control shell vibrations and, for instance, increase a zone of harmonic vibrations. It is worth noticing that the introduced changes of amplitude and frequency of the exciting load allowed to avoid stiff stability loss and to keep only harmonic shell vibrations. 
Finally, a package of programs has been developed allowing for the construction of a mathematical model of an elastic spherical shell. Large amounts of numerical experiments have been carried out, yielding new results in the field of stochastic vibrations of flexible shallow spherical shells.

\section{References}

[1] J. Awrejcewicz and V. A. Krysko, Feigenbaum scenario exhibited by thin plate dynamics, Nonlinear Dynamics 24 (2001), no. 4, 373-398.

[2] J. Awrejcewicz and A. V. Krysko, Analysis of complex parametric vibrations of plates and shells using Bubnov-Galerkin approach, Archive of Applied Mechanics 73 (2003), no. 7, 495-504.

[3] J. Awrejcewicz and V. A. Krysko, Nonclassical Thermoelastic Problems in Nonlinear Dynamics of Shells. Applications of the Bubnov-Galerkin and Finite Difference Numerical Methods, Scientific Computation, Springer, Berlin, 2003.

[4] __ Nonlinear coupled problems in dynamics of shells, International Journal of Engineering Science 41 (2003), no. 6, 587-607.

[5] J. Awrejcewicz, V. A. Krysko, and A. V. Krysko, Spatial-temporal chaos and solitons exhibited by von Kármán model, International Journal of Bifurcation and Chaos in Applied Sciences and Engineering 12 (2002), no. 7, 1465-1513.

[6] J. Awrejcewicz, V. A. Krysko, and A. V. Krysko, Complex parametric vibrations of flexible rectangular plates, Meccanica. International Journal of the Italian Association of Theoretical and Applied Mechanics 39 (2004), no. 3, 221-244.

[7] J. Awrejcewicz, V. A. Krysko, and G. G. Narkaitis, Bifurcations of a thin plate-strip excited transversally and axially, Nonlinear Dynamics 32 (2003), 187-209.

[8] J. Awrejcewicz, V. A. Krysko, and A. F. Vakakis, Nonlinear Dynamics of Continuous Elastic Systems, Springer, Berlin, 2004.

[9] V. F. Fedos'ev, Application of the step method to analyse stability of compressed rod, Prikladnaya Matematika i Mekhanika 27 (1963), no. 5, 833-841 (Russian).

[10] - On the method of solutions of stability of deformable bodies, Prikladnaya Matematika i Mekhanika 27 (1963), no. 2, 265-275 (Russian).

[11] M. J. Feigenbaum, The universal metric properties of nonlinear transformations, Journal of Statistical Physics 21 (1979), no. 6, 669-706.

[12] A. Hübler and E. Lüscher, Resonant stimulation and control of nonlinear oscillators, Naturwissenschaften 76 (1989), no. 2, 67-69.

[13] E. A. Jackson, The entrainment and migration controls of multiple-attractor systems, Physics Letters. A 151 (1990), no. 9, 478-484.

[14] - On the control of complex dynamic systems, Physica D. Nonlinear Phenomena 50 (1991), no. 3, 341-366.

[15] V. A. Krysko, J. Awrejcewicz, and V. M. Bruk, On the solution of a coupled thermo-mechanical problem for non-homogeneous Timoshenko-type shells, Journal of Mathematical Analysis and Applications 273 (2002), no. 2, 409-416.

[16] V. A. Krysko and I. V. Kravtsova, Stochastic vibrations of flexible flat axisymmetric shells exposed inhomogeneous loading, Proceedings of 7th International Conference on Dynamics of SystemTheory and Applications (Łódź, 2003), 2003, pp. 189-197.

[17] __ Stochastic vibrations of flexible axially symmetric supported along contour spherical shells, Izviestia VUZ, Maschinostroyeniye 1 (2004), 3-13 (Russian).

[18] V. A. Krysko and T. V. Shchekaturova, Chaotic vibrations of shallow shells, Izviesta AN Mekhanika Tviordoga Tela 4 (2004), 140-150 (Russian).

[19] L. D. Landau, On the problem of a turbulence, Doklady Akademii Nauk 44 (1944), no. 8, 339-342 (Russian). 
[20] B. B. Mandelbrot, The Fractal Geometry of Nature, Schriftenreihe für den Referenten, W. H. Freeman, California, 1982.

[21] P. Manneville and Y. Pomeau, Different ways to turbulence in dissipative dynamical systems, Physica D. Nonlinear Phenomena 1 (1980), no. 2, 219-226.

[22] E. Ott, C. Grebogi, and J. A. Yorke, Controlling chaos, Physical Review Letters 64 (1990), no. 11, 1196-1199.

[23] V. Petrov, V. Gaspar, J. Massere, and K. Showalter, Controlling chaos in the Belousov-Zhabotinsky reaction, Nature 361 (1993), no. 6409, 240-243.

[24] D. Ruelle and F. Takens, On the nature of turbulence, Communications in Mathematical Physics 20 (1971), 167-192.

[25] S. J. Schiff, K. Jerger, D. H. Duong, T. Chang, M. L. Spano, and W. L. Ditto, Controlling chaos in the brain, Nature 370 (1994), no. 6491, 615-620.

[26] T. Shinbrot, C. Grebogi, J. A. Yorke, and E. Ott, Using small perturbations to control chaos, Nature 363 (1993), no. 6428, 411-417.

[27] J. Singer, Y.-Z. Wang, and H. H. Bau, Controlling a chaotic system, Physical Review Letters 66 (1991), no. 9, 1123-1125.

[28] S. Smale, Dynamical systems and turbulence, Turbulence Seminar (Univ. Calif., Berkeley, Calif., 1976/1977), Lecture Notes in Math., vol. 615, Springer, Berlin, 1977, pp. 48-70.

[29] N. V. Valishvili, Methods of Computation of Rotational Shells, Mashinostroyeniye, Moscow, 1976.

J. Awrejcewicz: Department of Automatics and Biomechanics, Technical University of Lodz, 1/15 Stefanowskiego Street, 90924 Lodz, Poland

E-mail address: awrejcew@p.lodz.pl

V. A. Krysko: Department of Mathematics, Saratov State University, 410054 Saratov, Russia E-mail address: tak@san.ru

I. V. Kravtsova: Department of Mathematics, Saratov State University, 410054 Saratov, Russia

E-mail address: kravtsova@san.ru 


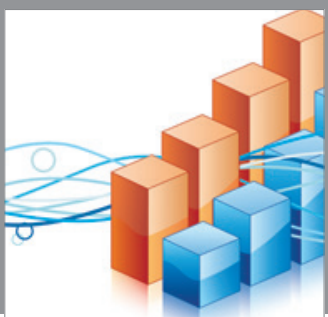

Advances in

Operations Research

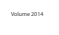

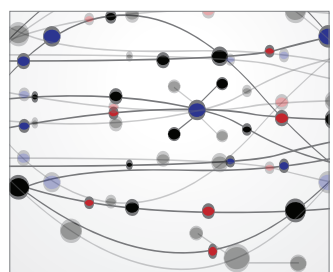

\section{The Scientific} World Journal
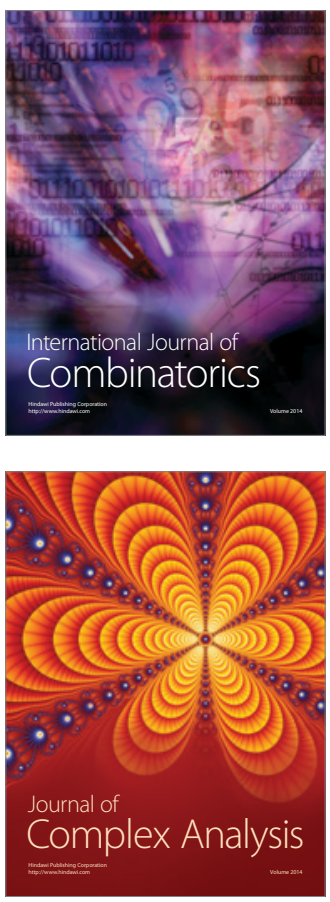

International Journal of

Mathematics and

Mathematical

Sciences
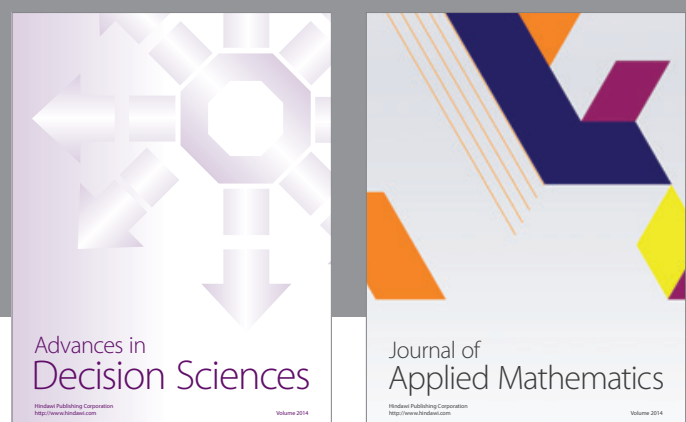

Journal of

Applied Mathematics
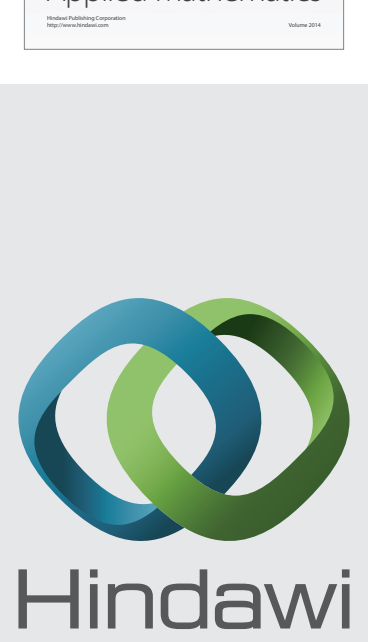

Submit your manuscripts at http://www.hindawi.com
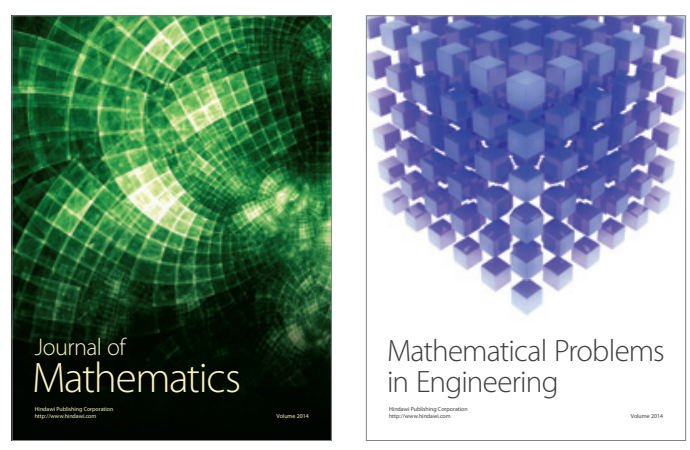

Mathematical Problems in Engineering
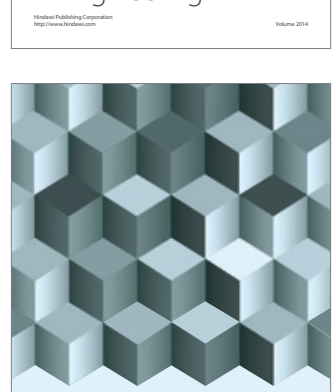

Journal of

Function Spaces
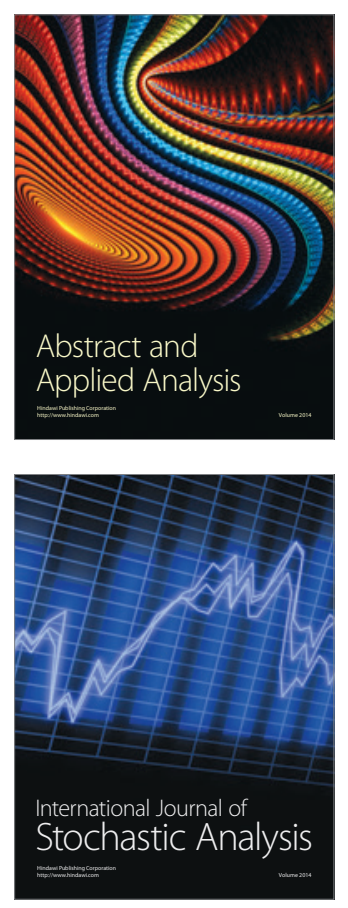

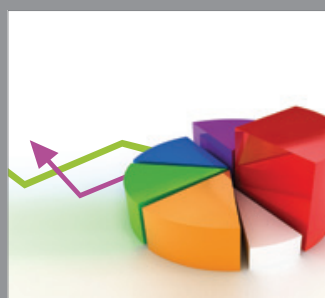

ournal of

Probability and Statistics

Promensencen
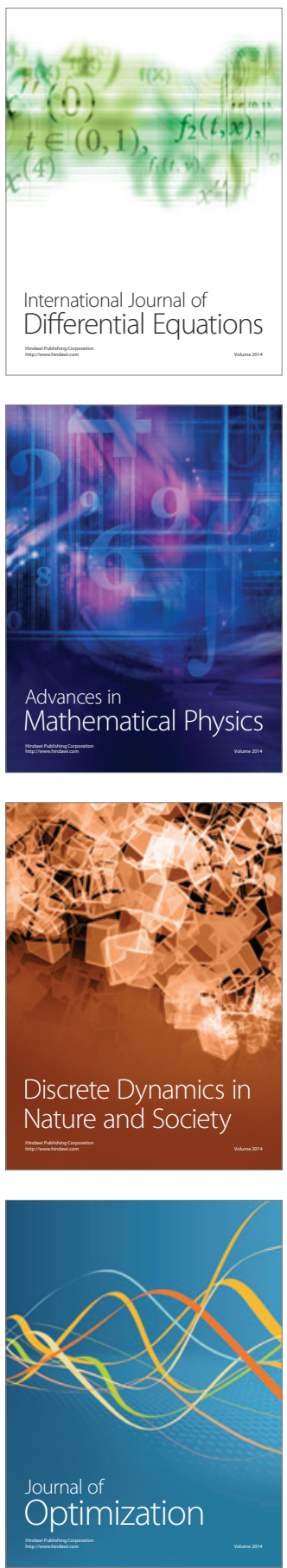